Rayleigh-Taylor and Richtmyer-Meshkov Instabilities and Mixing in Stratified Cylindrical Shells

K. O. Mikaelian

May 6, 2004

Physics of Fluids 
This document was prepared as an account of work sponsored by an agency of the United States Government. Neither the United States Government nor the University of California nor any of their employees, makes any warranty, express or implied, or assumes any legal liability or responsibility for the accuracy, completeness, or usefulness of any information, apparatus, product, or process disclosed, or represents that its use would not infringe privately owned rights. Reference herein to any specific commercial product, process, or service by trade name, trademark, manufacturer, or otherwise, does not necessarily constitute or imply its endorsement, recommendation, or favoring by the United States Government or the University of California. The views and opinions of authors expressed herein do not necessarily state or reflect those of the United States Government or the University of California, and shall not be used for advertising or product endorsement purposes. 


\title{
Rayleigh-Taylor and Richtmyer-Meshkov instabilities and mixing in stratified cylindrical shells
}

\author{
Karnig O. Mikaelian \\ University of California, \\ Lawrence Livermore National Laboratory \\ Livermore, California 94551
}

We study the linear stability of an arbitrary number $\mathrm{N}$ of cylindrical concentric shells undergoing a radial implosion or explosion.We derive the evolution equation for the perturbation $\eta_{i}$ at interface $\mathrm{i}$; it is coupled to the two adjacent interfaces via $\eta_{i \pm 1}$. For $\mathrm{N}=2$, where there is only one interface, we verify Bell's conjecture as to the form of the evolution equation for arbitrary $\rho_{1}$ and $\rho_{2}$, the fluid densities on either side of the interface. We obtain several analytic solutions for the $\mathrm{N}=2$ and 3 cases. We discuss freeze-out, a phenomenon that can occur in all three geometries (planar, cylindrical, or spherical), and "critical modes" that are stable for any implosion or explosion history and occur only in cylindrical or spherical geometries. We present numerical simulations of possible gelatin-ring experiments illustrating perturbation feedthrough from one interface to another. We also develop a simple model for the evolution of turbulent mix in cylindrical geometry and define a geometrical factor $G$ as the ratio $h_{\text {cylindrical }} / h_{\text {planar }}$ between cylindrical and planar mixing layers. We find that $G$ is a decreasing function of $R / R_{o}$, implying that in our model 
$h_{\text {cylindrical }}$ evolves faster (slower) than $h_{\text {planar }}$ during an implosion

(explosion).

\section{Introduction and Comparison}

Basic fluid flows occur in one of three geometries: planar, cylindrical, or spherical. When two (or more) fluids are present, the basic flow may be parallel or perpendicular to the interface(s) between the fluids. Perturbations to the basic flow evolve and generally grow with time leading to hydrodynamic instabilities. Well-known examples are the Kelvin-Helmholtz ${ }^{1,2}(\mathrm{KH})$ instability occurring in flows parallel to an interface, the Rayleigh-Taylor $^{3,4}$ (RT) instability, and the Richtmyer-Meshkov ${ }^{5,6}$ (RM) instability occurring in flows perpendicular to an interface. In this paper we study RT and RM instabilities in cylindrical geometry.

RT and RM instabilities appear in a variety of processes and physical scales: distribution of micro-organisms ${ }^{7}$, Inertial-Confinement-Fusion (ICF) capsules ${ }^{8}$, nuclear reactors ${ }^{9}$, supernova explosions ${ }^{10}$, etc. Our interest is primarily in ICF capsules and convergent hydrodynamic instabilities. Although ICF capsules are spheres, we will see that all convergence effects are captured in cylindrical geometry, albeit at reduced levels.

A number of experiments have been carried out with the same purpose of capturing convergence effects: electron-beam targets ${ }^{11}$, cylindrical metal shells ${ }^{12}$, laser $\operatorname{targets}^{13}$, and gelatin rings ${ }^{14,15}$. Numerical Euler simulations in cylindrical geometry have been performed with emphasis on high Mach-number flows ${ }^{16}$. To obtain analytic results, we first study incompressible, irrotational and inviscid flows with perturbations in the linear regime (Sec. II-IV), then present an analytic model for turbulence in cylindrical geometry 
(Sec. V), and finally we compare our analytic results with numerical simulations that cover nonlinear and compressible flows (Sec. VI). Conclusions are given in Sec. VII. In planar geometry, the RT instability evolves ${ }^{3,4}$ according to:

$$
\frac{d^{2} \eta}{d t^{2}}-g k A \eta=0
$$

where $\eta(t)$ is the amplitude of sinusoidal perturbations of wavelength $\lambda=\frac{2 \pi}{k}$ between fluids of densities $\rho_{1}$ and $\rho_{2}, A=\left(\rho_{2}-\rho_{1}\right) /\left(\rho_{2}+\rho_{1}\right)$ is the Atwood number, and $g$ is the acceleration directed from fluid 1 towards fluid 2. In cylindrical geometry we find:

$$
\frac{d^{2} \eta}{d t^{2}}+2 \frac{\dot{R}}{R} \frac{d \eta}{d t}-(n A-1) \frac{\ddot{R}}{R} \eta=0
$$

where $R$ is the cylindrical radius between fluids 1 and 2, and $n$ is the mode number of the perturbation. A "dot" over a letter refers to the time derivative. We will refer to Eq. (1b) as "Bell's equation" because, as far as we know, Bell ${ }^{17}$ was the first to consider the RT instability in cylindrical geometry (the RM instability was not discovered at that time). Bell derived the governing equations for two specific cases and made a conjecture as to the general form of that equation. We know of no earlier derivation of Eq. (1b), which indeed verifies that Bell's conjecture was correct. His two specific cases correspond to $A=+1$ and -1.

In spherical geometry, we have ${ }^{18}$ :

$$
\frac{d^{2} \eta}{d t^{2}}+3 \frac{\dot{R}}{R} \frac{d \eta}{d t}-n A(n) \frac{\ddot{R}}{R} \eta=0
$$

where $R$ is now the spherical radius between fluids 1 and 2 , and 


$$
n A(n)=\frac{n(n-1) \rho_{2}-(n+1)(n+2) \rho_{1}}{n \rho_{2}+(n+1) \rho_{1}}=\frac{2 n(n+1) A}{2 n+1-A}-2
$$

Eqs. (1a), (1b), and (1c) are listed in order of increasing convergence effects, and this is reflected in the increase of the coefficient in the $2^{\text {nd }}$ term of Eqs. (1b) and (1c): as we go from cylindrical geometry to the more convergent spherical geometry that factor increases from 2 to 3 . Note that there is no corresponding term in planar geometry, Eq. (1a). Of course, in the limit of large $R$ Eqs. (1b) and (1c) both reduce to Eq. (1a), the relationship between $\mathrm{k}, R$, and $\mathrm{n}$, being $k=n / R$. Although gravity and radial acceleration are not equivalent in convergent geometries, $\ddot{R}$ plays the role of gravity in Eqs. (1b) and (1c). Of course, unlike spherical geometry, cylindrical geometry has no physical object with a "constant" radial gravitational field, and our applications are to systems with moving radii such as the experiments in Refs. 11-15.

In the same order of increasing convergence effect we list the RM results:

$$
\begin{gathered}
\eta(t)=\eta(0)(1+k A \Delta \mathrm{v} t), \\
\eta(t)=\eta(0)\left[1+(n A-1)\left(1-R_{0} / R\right)\right], \\
\eta(t)=\eta(0)\left\{1+\frac{n A(n)}{2}\left[1-\left(\frac{R_{0}}{R}\right)^{2}\right]\right\} .
\end{gathered}
$$

In deriving Eqs. $(3 a-3 c)$ we assume that the interface moves with constant velocity after the "shock" which is represented as an impulsive acceleration, $g=\ddot{R}=\Delta \mathrm{v} \delta(t)$. This was done first by Richtmyer ${ }^{5}$ who gave Eq. (3a). Eq. (3b) is derived in the appendix of this 
paper, and Eq. (3c) was reported earlier ${ }^{19}$. Using $R=R_{0}+\Delta \mathrm{v} t$, we see that the latter two equations reduce to the first in the limit of large $R$.

If the velocity after the shock is not constant then the shock merely sets the initial conditions that one must use in solving Eqs. $(1 \mathrm{a}, \mathrm{b}, \mathrm{c})$ with the appropriate $R(t)$. See, for example, Eq. (A13) in the Appendix. We will illustrate with several examples.

In Sec. V, we consider a simple model for $\mathrm{h}(t)$, the turbulent mixing width in cylindrical geometry. To put it in context with the other two geometries, we again list the results of the model in increasing order of convergence effects:

$$
\begin{gathered}
\frac{d^{2} h}{d t^{2}}-c g A=0, \\
\frac{1}{R^{2}} \frac{d}{d t}\left(R^{2} \frac{d h}{d t}\right)-c A \ddot{R}=0 \\
\frac{1}{R^{3}} \frac{d}{d t}\left(R^{3} \frac{d h}{d t}\right)-c A \ddot{R}=0
\end{gathered}
$$

where $c$ is a constant.

Strictly speaking, one must use $c_{\text {planar }}, c_{\text {cylindrical }}$, and $c_{\text {spherical }}$ in Eqs.(4a) through (4c) respectively because $c$ may be different in each geometry. As discussed in Sec. V there is no computational or experimental evidence that a universal constant can describe turbulent mixing in all three geometries. However, since the three geometries rarely, if ever, co-exist, this is an issue of notation only at this stage. 
The above equations can be solved for any acceleration history $g(t)$ or $\ddot{R}(t)$. The simplest results are obtained for an impulsive acceleration:

$$
\begin{gathered}
h=c A \Delta \mathrm{v} t, \\
h=c A \Delta \mathrm{v} t R_{0} / R, \\
h=\frac{c A \Delta \mathrm{v} t R_{0}}{2 R}\left(1+R_{0} / R\right) .
\end{gathered}
$$

Unlike $\eta(t)$, which can be derived from first principles under the assumption of linearity and incompressibility, the equations for $h(t)$ are only the results of a model designed to capture the effect of convergence. More elaborate models, including dissipation effects are available ${ }^{20}$.

RT and RM instabilities in an arbitrary number of spherical shells were studied in Ref. 19; in this paper we do the same for cylindrical shells. Several phenomena that arise only in the presence of convergence were reported in Ref. 19. The overreaching conclusion of the present paper is that each and every phenomenon that occurs in spherical geometry occurs also in cylindrical geometry. From the experimental point of view, cylindrical geometry offers ease of diagnostics, although recent experiments have observed some convergence effects in imploding spheres ${ }^{21}$. Our results establish a one-toone correspondence between the two geometries, and for that purpose we will use the same notation and terminology as in Ref. 19.

We close this Section with a brief discussion of earlier work in plane geometry. The RT instability in an arbitrary number of incompressible fluid layers was studied in Ref. 
22. The effect of surface tension was added in Ref. 23, and compressibility in Ref. 24. Complementing the work of Richtmyer, ${ }^{5}$ who considered only the case where a shock is reflected from the interface between two semi-infinite fluids, Yang, Zhang and Sharp ${ }^{25}$ considered the case where a rarefaction is reflected from the interface, which occurs when a shock moves from a heavy into a light fluid. Recent experiments on the RM instability covering the linear as well as the nonlinear regime are reported by Jacobs et al. ${ }^{26}$ Explicit expressions for $\eta(t)$ in these two regimes can be found in Ref. 27. For a review of RT and RM instabilities see Sharp, ${ }^{28} \mathrm{Kull}^{29}$ and, more recently, Brouillette. ${ }^{30}$

\section{General Evolution Equations}

We consider a system of $N$ cylindrical coaxial shells: The first fluid, of density $\rho_{1}$, extends between $R=0$ and $r=R_{1}$, and the last fluid of density $\rho_{N}$ extends from $r=R_{N-1}$ to infinity. In between, region $i$ consists of a fluid of density $\rho_{i}$ extending between $R_{i-1}$ and $R_{i}, i=1,2, \ldots, \mathrm{N}$ with the understanding that $R_{0}=0, R_{N}=\infty$, and that the first and last regions need special treatment. We are considering only radial incompressible flow.

As Plesset noted for the spherical case ${ }^{18}$, we must assume that if $\rho_{1} \neq 0$ then a source or a sink exists at $r=0$ to allow that region to expand or contract while maintaining a constant density.

Perturbations are introduced by taking the interface $r_{i}$ between $\rho_{i}$ and $\rho_{i+1}$, nominally at $R_{i}$, to be given by

$$
r_{i}=R_{i}+\eta_{i} \cos n \theta
$$


where $R_{i}=R_{i}(t)$ and $\eta_{i}=\eta_{i}(t)$, but mode number $n$ and angle $\theta$ (relative to some arbitrary axis) are not functions of time.

In region $i$ of density $\rho_{i}$ and extending between $r_{i-1}$ and $r_{i}$ we introduce a velocity potential $\varphi_{i}$ such that $\dot{r}=-\partial \varphi_{i} / \partial r$ in that region. For incompressible flow $\nabla^{2} \varphi_{i}=0$ and therefore

$$
\varphi_{i}=-R_{i} \dot{R}_{i} \ln r+B_{i} r^{n} \cos n \theta+C_{i} r^{-n} \cos n \theta
$$

where the first term gives the basic flow and the second and third terms are small (in a sense clarified below) perturbations to it. $B_{i}$ and $C_{i}$ are "constants" in the sense that they are independent of $r$. They are functions of time and belong to region $i$ as indicated by their subscript. The special treatments in the first and last regions call for $C_{1}=B_{N}=0$. As in spherical geometry, conservation of mass implies that

$$
R_{i}^{2}(t)-R_{j}^{2}(t)=\text { constant }=R_{i}^{2}(0)-R_{j}^{2}(0)
$$

so that specifying the basic flow at any one interface automatically specifies the other interfaces-in other words Eq. (8) implies that $R_{i} \dot{R}_{i}=R_{j} \dot{R}_{j}$ and hence the first term in Eq. (7) is actually independent of $i$, just like the term $R_{i}^{2} \dot{R}_{i}$ in spherical geometry.

Since the derivations are very similar to the spherical case, we will only give the final result and then outline the steps. The basic flow is given by

$$
P_{i}(t)-P_{i+1}(t)=\left(\rho_{i}-\rho_{i+1}\left[\frac{1}{2} \dot{R}_{i}^{2}+\left(\ln R_{i}\right) \frac{d}{d t}\left(R_{i} \dot{R}_{i}\right)\right],\right.
$$

and the general evolution equation for the perturbation amplitude $\eta_{i}(t)$ is given by 


$$
\left(\rho_{i+1}-\rho_{i}\right) \frac{d}{d t}\left(R_{i} \dot{R}_{i} \eta_{i}\right)=R_{i}^{n+1}\left[\rho_{i+1} \frac{d B_{i+1}}{d t}-\rho_{i} \frac{d B_{i}}{d t}\right]+(n \rightarrow-n)
$$

In Eq. (9) $\mathrm{P}_{i, i+1}(t)$ are Bernoulli "constants" and in Eq. (10) $B_{i}$ is given by

$$
B_{i}=\frac{1}{n\left[R_{i}^{2 n}-\left(R_{i-1}\right)^{2 n}\right]}\left[R_{i-1}^{n} \frac{d}{d t}\left(R_{i-1} \eta_{i-1}\right)-R_{i}^{n} \frac{d}{d t}\left(R_{i} \eta_{i}\right)\right] .
$$

Eq. (10) is a second order differential equation in $\eta_{i}(t)$ and, as in planar and spherical geometry, exhibits interface coupling because it involves $\eta_{i-1}, \eta_{i}$, and $\eta_{i+1}$. It is important to note that the terms denoted by $(n \rightarrow-n)$ arise from the corresponding $C_{i}$ terms, i.e., they stand for $\left(R_{i}\right)^{1-n}\left[\rho_{i+1} d C_{i+1} / d t-\rho_{i} d C_{i} / d t\right]$, and $C_{i}=B_{i}(n \rightarrow-n)$. The reason this is important is that we have to set $C_{1}=B_{N}=0$.

We briefly outline the steps leading to Eqs. (9) and (10) that parallel the spherical case [compare with Eqs. (19) and (20) in Ref. 19]: $\dot{r}=\dot{R}+\dot{\eta} \cos n \theta=-\partial \varphi / \partial r$ is applied twice: at $r=r_{i-1}$ and $r_{i}$, i.e., at the two interfaces on each side of region $i$, and the results expanded in $\eta_{i, i-1}$ keeping only the linear terms. For this expansion to be valid, one must have $\left|n \eta_{i}\right|<<R_{i}$. These two equations are solved for $B_{i}$ and $C_{i}$. The results are substituted in the Bernoulli equation which is also expanded keeping only the lowest order terms (what we have called basic flow) and terms linear in $\eta$, the latter all being proportional to $\cos n \theta$. The lowest order terms yield the basic flow, Eq. (9), and the linear terms yield Eq. (10) after dropping the $\operatorname{common}$ factor $\cos n \theta$.

Eq. (10) shows that the evolution is symmetric under $n \rightarrow-n$. This is true, however, only when $\rho_{1}=\rho_{N}=0$ and we do not have to implement the special treatments in the first 
and last regions calling for $C_{1}=B_{N}=0$. If $\rho_{1} \neq 0$ and/or $\rho_{N} \neq 0$ then the $n \rightarrow-n$ symmetry is broken as seen, for example, in Bell's equation, Eq. (1b).

\section{III. $\mathbf{N}=2$ Case}

We must set $C_{1}=B_{2}=0$ in Eq. (10) and, since there is only one radius and one perturbation amplitude, we simplify our notation by using $R=R_{1}, \eta=\eta_{1}$, to get

$$
\left(\rho_{2}-\rho_{1}\right) \frac{d}{d t}(R \dot{R} \eta)=R^{n+1}\left[-\rho_{1} \frac{d B_{1}}{d t}\right]+R^{1-n}\left[\rho_{2} \frac{d C_{2}}{d t}\right] .
$$

From Eq. (11) we have

$$
B_{1}=-\frac{R^{-n}}{n} \frac{d}{d t}(R \eta)
$$

and

$$
C_{2}=\frac{R^{n}}{n} \frac{d}{d t}(R \eta)
$$

which, when substituted in Eq. (12), lead to what we have called "Bell's equation":

$$
\frac{d^{2} \eta}{d t^{2}}+\frac{2 \dot{R}}{R} \frac{d \eta}{d t}-(n A-1) \frac{\ddot{R}}{R} \eta=0 .
$$

Alternative forms of this equation are:

$$
\frac{\ddot{e}}{e}=n A \frac{\ddot{R}}{R}, e \equiv \eta R,
$$


and

$$
\frac{1}{R^{2}} \frac{d}{d t}\left(R^{2} \frac{d \eta}{d t}\right)-(n A-1) \frac{\ddot{R}}{R} \eta=0
$$

As a historical remark, Bell derived Eq. (15) for the cases $A=+1$ and -1 obtaining $\pm n \ddot{R} / R$ for the right-hand-side( $r h s)$ of that equation, and conjectured that in the general case it would be $n A \ddot{R} / R$, which we have now verified. It is interesting that he also did the spherical geometry for the same two cases $A= \pm 1$, but this time he did not venture any conjecture as to its general form. Plesset was the first to derive the general $-A$ equation in spherical geometry, and its $A$-dependence [see Eqs. (1c) and (2)] indeed defies simple conjecture.

In the Introduction, we mentioned that the types of solutions we find here for Bell's equation match exactly those we found earlier for Plesset's equation, so we will be brief. We have two class- $A$ solutions which are valid for arbitrary $R(t)$, but require specific values of $n A$ (note that only this combination appears in Bell's equation.) The first applies when $n A=0$ for which Eq. (15) gives $\dot{e}=$ constant and the solution is

$$
\eta(t)=\eta_{0} \frac{R_{0}}{R}+\left(\dot{\eta}_{0} R_{0}+\eta_{0} \dot{R}_{0}\right) t / R
$$

Note that perturbations of any mode $n$ evolve with time even when $\rho_{1}=\rho_{2}(A=0)$. This is purely a convergent effect, and the same was found in spherical geometry ${ }^{19}$.

The second class-A solution brings out what we have called "critical modes". It is obtained when $n A=1$ for which Eq. (16) gives $R^{2} \dot{\eta}=$ constant, and the solution is 


$$
\eta(t)=\eta_{0}+\dot{\eta}_{0} R_{0}^{2} \int \frac{d t}{R^{2}}
$$

(Zero subscripts refer to $t=0$ values). These modes are interesting because for any velocity history $R(t)$ if $\dot{\eta}_{0}=0$, as is often the case, then these modes do not grow at all. In these cases, the inertial forces that arise because $\rho_{1} \neq \rho_{2}$ are canceled exactly by the convergence effect. This is a phenomenon that does not occur in plane geometry, but of course it occurs in spherical geometry where it was first reported ${ }^{19}$. Since $n$ must be an integer, critical modes occur only for specific values of $\rho_{2} / \rho_{1}$ given by the $n A=1$ condition. For example, the $n=4$ mode will be a critical mode for $A=1 / 4\left(\rho_{2} / \rho_{1}=5 / 3\right)$ in cylindrical geometry. In spherical geometry where the condition reads $n A(n)=0$, this would require $A=3 / 7\left(\rho_{2} / \rho_{1}=5 / 2\right)$.

We now turn to class-B solutions, which are valid for arbitrary $n A$, but require specific implosion or explosion history $R(t)$. We have found only four such solutions and they are given in the Appendix. The simplest but perhaps the most interesting solution is for the case of shocks preceded or followed by constant velocities. The result for an isolated shock is Eq. (3b). A more general solution and an extended discussion is given in the Appendix.

\section{IV. $\mathbf{N}=3$ Case}

Applying the general evolution equation, Eq. (10), for $i=1$ and $i=2$ we obtain two coupled second-order differential equations for $\eta_{1,2}(t)$. We have written a simple computer program that solves these equations, testing the program by verifying that large- 
n-perturbations obey Bell's equation at each interface. Results will be given in Section VI. Some simplification is obtained by considering Taylor's case, $\rho_{1}=\rho_{3}=0$, i.e., a cylindrical fluid of any density in the region $R_{1} \leq r \leq R_{2}$ and no inner or outer fluid. Substantial simplification, however, is achieved only for the case of a thin shell, i.e., for $\Delta R<<R$ where, following Ref. 19, we define

$$
\Delta R=R_{2}-R_{1}, R=\left(R_{1}+R_{2}\right) / 2
$$

The result can be written succinctly if we define the variables $\Delta \eta$ and $\eta$ :

$$
\Delta \eta=\eta_{2}-\eta_{1}, \eta=\left(\eta_{1}+\eta_{2}\right) / 2
$$

After a lengthy algebra we find:

$$
\frac{d^{2} \eta}{d t^{2}}=-\ddot{R} \frac{\Delta \eta}{\Delta R}
$$

and

$$
\frac{d^{2}}{d t^{2}}\left(\frac{R \Delta \eta}{\Delta R}\right)=\ddot{R}\left[2 \frac{\Delta \eta}{\Delta R}-\left(n^{2}-1\right) \eta / R\right]
$$

which can be compared with Eqs. (44a,b) in Ref. 19.

Since in Taylor's case the first and last densities vanish, i.e., $\rho_{1}=\rho_{N}=\rho_{3}=0$, we explicitly verify that Eqs. (20 a,b) are indeed invariant under $n \rightarrow-n$ (see the discussion at the end of Sect. II).

Eqs. $(20 \mathrm{a}, \mathrm{b})$ can be solved for the case of a "shock", i.e., impulsive acceleration, followed by a constant radial velocity: Let 


$$
\ddot{R}=\Delta \mathrm{v} \delta(t)=\left(R_{0} / T\right) \delta(t) .
$$

Throughout this paper a positive (negative) time constant $\mathrm{T}$ will imply explosion (implosion). Assuming an initially stationary shell, i.e., $\dot{R}\left(0_{-}\right)=\dot{\eta}\left(0_{-}\right)=\Delta \dot{\eta}\left(0_{-}\right)=0$, we have, immediately after shock passage,

$$
\dot{R}\left(0_{+}\right)=\Delta \mathrm{v}=R_{0} / T
$$

obtained by integrating Eq. (21). Substituting Eq. (21) in Eq. (20a) and integrating over $0_{-} \leq t \leq 0_{+}$only we obtain

$$
\dot{\eta}\left(0_{+}\right)=-\Delta \mathrm{v} \frac{\Delta \eta(0)}{\Delta R(0)} .
$$

The same substitution and integration in Eq. (20b) leads to

$$
\frac{d}{d t}\left(\frac{R \Delta \eta}{\Delta R}\right)_{0_{+}}=\Delta \mathrm{v}\left[\frac{2 \Delta \eta(0)}{\Delta R(0)}-\left(n^{2}-1\right) \eta(0) / R(0)\right\rceil .
$$

To clarify, $R(0)$ is the same as $R_{0}, \eta(0)=\eta_{0}, \Delta \eta(0)=\Delta \eta_{0}$, etc. Substituting $\Delta \mathrm{v}=R_{0} / T$ in Eq. (23a), we have

$$
\dot{\eta}\left(0_{+}\right)=-\frac{R_{0}}{T} \frac{\Delta \eta_{0}}{\Delta R_{0}} .
$$

Using Eq. (22) and the relations $\Delta \dot{R}=-\Delta R \dot{R} / R$ in Eq. (23b) we find

$$
\Delta \dot{\eta}\left(0_{+}\right)=-\left(n^{2}-1\right) \Delta \mathrm{v} \eta_{0} \frac{\Delta R_{0}}{R_{0}^{2}}=-\left(n^{2}-1\right) \eta_{0} \frac{\Delta R_{0}}{T R_{0}} .
$$


Eqs. (24a) and (24b) give the initial conditions immediately after the passage of the shock. We now return to the two basic equations, Eqs. (20a) and (20b), and solve them for the next stage, i.e., after shock passage, where now $\ddot{R}=0$ and they can be solved in a simple manner using Eqs. (24a,b) as initial conditions. The result is

$$
\eta(t)=\eta(0)-\Delta \eta_{0} \frac{\Delta \mathrm{v}}{\Delta R_{0}} t=\eta(0)-\Delta \eta_{0} t / T
$$

and

$$
\begin{aligned}
\Delta \eta(t) & =\frac{\Delta \eta_{0}(1+2 t / T)+\Delta \dot{\eta}\left(0_{+}\right) t}{(1+t / T)^{2}} \\
& =\frac{\Delta \eta_{0}(1+2 t / T)-\left(n^{2}-1\right) \eta_{0} \frac{\Delta \mathrm{v} \Delta R_{0}}{R_{0}^{2}} t}{(1+t / T)^{2}} \\
& =\frac{\Delta \eta_{0}(1+2 t / T)-\left(n^{2}-1\right) \eta_{0} \frac{\Delta R_{0}}{R_{0}} \frac{t}{T}}{(1+t / T)^{2}} .
\end{aligned}
$$

It is remarkable that Eq. (25a) is identical to the spherical case (Eq. (47a) in Ref. (19)) and, in fact, identical to the planar case also ${ }^{31}$. Note that it is independent of mode number $n$. Eq. (25b) is slightly different in spherical geometry—compare with Eq. (47b) in Ref. 19. In the limit $n \rightarrow \infty, R \rightarrow \infty, n / R \rightarrow k$, both cylindrical and spherical geometries reduce to the planar limit which is ${ }^{31}$

$$
\Delta \eta(t)=\Delta \eta_{0}-\eta_{0} \Delta \mathrm{v} \Delta R_{0} k^{2} t .
$$

By solving numerically the coupled differential equations for arbitrary $\rho_{1}, \rho_{2}, \rho_{3}, R_{1}, R_{2}$, and $n$, we have verified that for large-n perturbations the interfaces 
decouple so that one can apply Bell's equation at $R_{1}$ and $R_{2}$ independently. Similarly if $R_{2}-R_{1}>>R_{1}$, i.e., for thick shells, or if $\rho_{2}=0$. In general, since implosions (explosions) cause a shell to thicken-up (thin-out), interface coupling or feedthrough decreases (increases) in converging (diverging) flows. This behavior is the same in cylindrical as it was in spherical geometry. ${ }^{19}$

Consider a cylindrical shell with $R_{1}(0)=25$ and $R_{2}(0)=26$ (the units are immaterial only ratios are relevant). It implodes in 8 units of time to a final $R_{1}(8)=1$. By mass conservation, $R_{2}=\left(R_{1}^{2}+R_{2}^{2}(0)-R_{1}^{2}(0)\right)^{1 / 2}=\left(1^{2}+26^{2}-25^{2}\right)^{1 / 2} \approx 7.2$ at $t=8$. The same example was considered in spherical geometry ${ }^{19}$ where $R_{2}=\left(R_{1}^{3}+R_{2}^{3}(0)-R_{1}^{3}(0)\right)^{1 / 3}$ $=\left(1^{3}+26^{3}-25^{3}\right)^{1 / 3} \approx 12.5$. In other words the shell thickens up from an initial value of 1 to a final thickness of 6.2 in cylindrical geometry compared with 11.5 in spherical geometry. Clearly, in the early stages when the shell is thin perturbations will readily feed through from one surface to another, but in the later stages as the shell thickens up the interfaces decouple and the perturbation at each surface grows independently of the other.

The evolution of the perturbations depends on the implosion history. We illustrate with two different implosions, shown in Fig. 1(a) and Fig. 2(a), of the same cylindrical shell with the same initial $\left(R_{1}(0)=25, R_{2}(0)=26\right)$ and final $\left(R_{1}(8)=1, R_{2}(8) \approx 7.2\right)$ configurations. The first, Fig. 1(a), is the "accel/decel" implosion studied earlier" $\ddot{R}_{1}=-1.5$ for $0<t<4$ and $\ddot{R}_{1}=+1.5$ for $4<t<8$. One can easily verify that this leads to $R_{1}(8)=1$. The second, Fig. 2(a), is a "shock" implosion, $\ddot{R}=\Delta \mathrm{v} \delta(t)$, as in Eq. (21). To obtain $R_{1}(8)=1$, we set $\Delta \mathrm{v}=-\left(25.5-\frac{1+\sqrt{1+51}}{2}\right) / 8 \approx-2.7$. 
We should clarify that by "constant" velocity we mean a time-independent velocity at one radius only (the average radius $\mathrm{R}$ in the example shown in Fig. 2, or, for Eq. (3), the interface between two fluids). As we discussed following Eq. (8) expressing mass conservation of incompressible fluids, the velocity throughout the fluid is radiusdependent through $R_{i} \dot{R}_{i}=R_{j} \dot{R}_{j}$ so that the velocity is inversely proportional to the radius.

The evolution of the perturbations can be calculated in one of four ways. The simplest is to apply Bell's equation at each interface. Since feedthrough is ignored in this approach, one can get completely wrong results (we will not show them), particularly when an initial amplitude vanishes. It remains zero, according to Bell's equation, while in fact feedthrough from the other interface induces a finite amplitude possibly growing very large at the initially smooth surface.

The exact solution is obtained by solving Eq. (10). The results are shown as solid lines in Fig. 1(b) and Fig. 2(b) for the "accel/decel" and "shock" implosions respectively. We have chosen $\rho_{1}=\rho_{3}=0, n=10$, and $\eta_{1}(0)=\eta_{2}(0)=1$, (the units here are immaterial for a different reason: The theory is linear). These figures show how perturbations evolve differently with different implosion histories even though the initial and final configurations are the same in both types of implosion. The "accel/decel" implosion is clearly much more susceptible to perturbation growth. We found the same to be true for other values of $n$ or $\eta_{1,2}(0)$.

The third way is to solve the thin-shell equations, Eqs.( 20a) and (20b). The results are shown by the dashed lines in Fig. 1(b). They are surprisingly good until $t \approx 5$ when they start deviating substantially from the exact results. Clearly the shell cannot be treated as "thin" after this time. Actually, the shell has not yet thickened-up much, but $R$ has 
decreased to $\sim 9$, and the condition for validity reads $\Delta R / R<<1$, good at $t=0$ when $\Delta R / R=1 / 25.5$ but not after $\mathrm{t}>5$ when $\Delta R / R>0.3$.

The fourth way is to use the analytic results, Eqs. (25a) and (25b), as shown by the dashed lines in Fig. 2(b). The same result is obtained by solving Eqs. (20a) and (20b) numerically, serving as a check of our numerical algorithm. While Eqs. (20a,b) are valid for any implosion (or explosion) history, Eqs. (25a,b) are valid only for a "shock”, Eq. (21), with a negative (or positive) $\Delta \mathrm{v}$. We find, again, that the thin-shell approximation breaks down after $t>5$.

Note that although the average radius $R$ is moving at constant velocity throughout the whole implosion, $R_{1}$ accelerates inward and $R_{2}$ accelerates outward, particularly at late times. In and of themselves both motions are "stable" in the RT sense: dense material $\left(\rho_{2}\right)$ accelerating into "vacuum" $\left(\rho_{1}=\rho_{3}=0\right)$. The well-known oscillations of stable gravity waves exhibit themselves at late times, $t>7$, when both perturbations $\eta_{1}$ and $\eta_{2}$ begin a phase-reversal in the exact calculation.

Eq. (25a) implies that if $\eta(0)=0\left(\eta_{10}=-\eta_{20}\right.$, i.e. "varicose" perturbations $)$ then $\eta(t) \sim t$. If $\Delta \eta(0)=0 \quad\left(\eta_{10}=\eta_{20}\right.$, i.e., "sinuous" perturbations $)$ then $\eta(t) \sim \eta(0)$ is constant. This is the example shown in Fig. 2(b) where the dashed lines $\eta_{1,2}$ grow large with opposite signs but their sum remains constant. Although valid for thin shells and $\rho_{1}=\rho_{3}=0$ only, Eqs. (25a,b) are the only explicit two-interface analytic solutions known to us. The same is true of spherical geometry - only thin RM solutions are known. ${ }^{19}$ The situation is different in plane geometry where one need not assume thin shells and the RM solutions have been reported ${ }^{31}$ for arbitrary thicknesses. Of course the first two-interface 
result was obtained by Taylor ${ }^{4}$ for the RT instability and for that reason we refer to the $\rho_{1}=\rho_{3}=0$ case as "Taylor's case" in any geometry or instability.

\section{A Model for Turbulent Mix in Cylindrical Geometry}

The presence of the dimensionless variable $R / R_{0}$ complicates matters in both spherical and cylindrical geometries when we try to develop a model for turbulent mix. Our approach in the spherical case was to start with Plesset's equation and take the limit

$$
n \rightarrow \infty, \eta \rightarrow 0, n \eta / R \rightarrow c=\text { const }
$$

to derive (see Eq. (50) in Ref. 19)

$$
\frac{1}{R^{3}} \frac{d}{d t}\left[R^{3} \frac{d h}{d t}\right]-c A \ddot{R}=0(\text { spherical })
$$

where $h$ is now interpreted as the turbulent mixing width into the heavier fluid.

If we apply the same methodology to Bell's equation in the form of Eq. (16), we obtain

$$
\left.\frac{1}{R^{2}} \frac{d}{d t}\left[R^{2} \frac{d h}{d t}\right]-c A \ddot{R}=0 \text { (cylindrical }\right)
$$

For any given implosion or explosion history $R(t)$ the solution to this equation can be found:

$$
h(t)=h(0)+R_{0}^{2} \frac{d h_{0}}{d t} \int_{0}^{t} \frac{d t}{R^{2}}+c A \int_{0}^{t}\left[\frac{1}{R^{2}} \int_{0}^{t} R^{2} \ddot{R} d t^{\prime}\right] d t .
$$


We shall limit to the $R T$ and $R M$ cases and assume $h(0)=d h_{0} / d t=0$. For the $R T$ case, we take $R=R_{0}+\frac{1}{2} g t^{2}$, where $g$ is a constant acceleration, and find:

$$
h=\frac{c A g t^{2}}{15}\left\{\frac{3}{2}+\frac{4 R_{0}}{R}+\frac{2 R_{0}}{R-R_{0}} \ln \left(R / R_{0}\right)\right\} \text {. }
$$

For the $R M$ case we take $\ddot{R}=\Delta \mathrm{v} \delta(t), R=R_{0}+\Delta \mathrm{v} t$, and find

$$
h=\frac{c A \Delta \mathrm{v} t R_{0}}{R} .
$$

The planar limits can be obtained by letting $R / R_{0} \rightarrow 1$ in the above equations yielding ${ }^{19}$

$$
\begin{aligned}
& h_{\text {planar }}=\frac{1}{2} c A g t^{2}, R T, \\
& h_{\text {planar }}=c A \Delta \mathrm{v} t, R M .
\end{aligned}
$$

Defining the geometrical factor $G$ as the ratio between $h_{c y l i n d r i c a l}$ and $h_{\text {planar }}$, we have

$$
G=\left[3+8 \frac{R_{0}}{R}+\frac{4 R_{0}}{R-R_{0}} \ln \left(R / R_{0}\right)\right\rceil / 15,
$$

for a constant acceleration, and

$$
G=\frac{R_{0}}{R}
$$

for a shock, to be compared with the spherical geometrical factors given in Ref. 19 (Eqs. 60 and 61). 
As expected, Eqs. (35) and (36) imply that for an implosion (explosion) the mixing width is larger (smaller) than the similarly driven planar case, i.e. $G>1(G<1)$ for an implosion (explosion). For the very late $\left(R / R_{0} \rightarrow \infty\right)$ stages of an acceleration-driven explosion Eq. (35) predicts $G \rightarrow 1 / 5$, to be compared with $1 / 7$ in spherical geometry ${ }^{19}$. For a shock-driven explosion $G \rightarrow 0$ but the mixing width $h$ approaches a constant: using $\Delta \mathrm{v} t=R-R_{0}$ in Eq. (32) we get $h \rightarrow c A R_{0}$, to be compared with $c A R_{0} / 2$ in spherical geometry ${ }^{19}$.

In this highly simplified approach we have only one constant, $c$, determining the evolution of mix in planar, cylindrical, or spherical geometry and for arbitrary acceleration histories. In the Introduction we discussed the possibility of using different constants in different geometries, in which case the above geometrical factors should be multiplied by $c_{c y l i n d r i c a l} / \mathrm{c}_{\text {planar }}$. While this may decrease the predictive power of our model the dependence on convergence, i.e., on $R / R_{0}$, is a non-trivial feature of the above geometrical factors. There is no dependence on initial conditions other than a simple additive constant $h_{0}$. As far as we know there have been no direct numerical simulations (DNS) of turbulence in cylindrical or spherical geometries. In planar geometry the DNS of Cook and Dimotakis ${ }^{32}$ find that the evolution of the RT mixing layer depends on initial conditions suggesting that $\mathrm{c}_{\text {planar }}$ may depend on initial perturbation wavelengths and amplitudes. On the other hand a variety of $R T$ experiments ${ }^{33,34}$ with different initial conditions indicate a fairly constant $\mathrm{c}$ in the range $c \approx 0.10-0.14$. For the $R M$ case the impulsively driven linear electric motor experiments [Dimonte and Schneider, Ref. 34] do not exhibit growth linear with time, while shock tube experiments ${ }^{35}$ are consistent with $h=c A \Delta \mathrm{v} t$ and $c \approx 0.10-0.14$. The situation is even more confusing with the few 
mix experiments done in cylindrical ${ }^{36}$ and spherical ${ }^{37}$ geometry, both carried out in shock tubes. Our presentation of an all-encompassing yet minimal model is motivated more by its simplicity than by any detailed comparison with experiments, many more of which are clearly needed to clarify the mix generated by $R T$ and $R M$ instabilities in various geometries.

\section{Numerical Simulations}

We now turn to $2 \mathrm{D} \mathrm{CALE}^{38}$ simulations of gelatin-ring experiments ${ }^{15}$. In those experiments gelatin rings of various thicknesses with perturbations on their outer surfaces were imploded and photographed. Preliminary results from CALE simulations were reported earlier ${ }^{39}$. Here we consider the following configuration: $\rho_{1}=\rho_{3}=1.2 \times 10^{-3} \mathrm{~g} / \mathrm{cm}^{3}$ for air at atmospheric pressure and temperature and the oxygen-acetylene mixture, the driving gas, $\rho_{2}=1.0 \mathrm{~g} / \mathrm{cm}^{3}$ for the gelatin ring. $R_{1}=4 \mathrm{~cm}, R_{2}=5.5 \mathrm{~cm}$. This $1.5 \mathrm{~cm}$ thick gelatin ring is inside a $10 \mathrm{~cm}$-radius containment fixture. The problem is initiated by sourcing $6.15 \mathrm{~kJ} / \mathrm{g}$ of energy into the driving gas region over a $10 \mu \mathrm{s}$ period, bringing the pressure up to $16.5 \mathrm{~atm}$. The gelatin-ring implodes compressing the air trapped in the center and, at about $770 \mu s$, bounces off the compressed ( 100 fold) air and moves back out. This motion, which is consistent with the experimental observations of $R_{1,2}(t)$, is shown in Fig. 3. The reverberations in $\dot{R}_{i}$ and $\ddot{R}_{i}$ are the results of sound waves captured in the compressible hydrocode CALE. We note that in contrast to planar layers where only one quantity (acceleration) controls the evolution of perturbations, in cylindrical geometry all six quantities $R_{1,2}, \dot{R}_{1,2}$, and $\ddot{R}_{1,2}$ enter in Eq. (10). 
Over the $1000 \mu \mathrm{s}$ of implosion and bounce the density $\rho_{3}$ of the driving gas changes by less than $19 \%$. The density $\rho_{2}$ of the gelatin ring, using an equation-of-state (EOS) for water, changes by less than $2 \%$. The density $\rho_{1}$ of the trapped central air, on the other hand, increases 100-fold, as mentioned above, because $R_{1}$ decreases tenfold from $4 \mathrm{~cm}$ down to $\sim 4 \mathrm{~mm}$. We did two sets of calculations: One in which we used a constant $\rho_{1}=\rho_{1}(0)$ in Eq. (10), and another using $\rho_{1}(t)$. The results differed by less than 5\%, probably because the density of 100 -fold compressed air, $0.12 \mathrm{~g} / \mathrm{cm}^{3}$, is still much less than the density of the gelatin ring at $1 \mathrm{~g} / \mathrm{cm}^{3}$. At higher compressions one must modify the perturbation equations as has been done in spherical geometry ${ }^{40}$.

A comparison with the experimental results was presented in Ref. 39 and will not be repeated here. Instead, we focus on four different configurations with emphasis on where the solutions to Eq. (10), which are linear and incompressible, agree or disagree with the fully nonlinear and compressible CALE simulations. The four cases all have $n=6$ and are distinguished by the initial values $\left(\eta_{1}(0), \eta_{2}(0)\right)$ measured in millimeters, and are $(0,0.5),(0.5,0),(0.5,0.5)$, and $(-0.5,0.5)$ for cases (a), (b), (c), and (d) respectively. In the nonlinear simulations bubbles of air and spikes of gelatin evolve differently at late times, particularly at the inner surface. We will display both bubble and spike amplitudes and compare them with the linear coupled calculation based on Eq. (10). Note that for this linear theory cases (c) and (d) are merely the sum and difference, respectively, of cases (a) and (b), but of course the nonlinear results are not related so simply.

The evolution of the amplitudes are shown in Figs. 4 thru 7 for cases (a) thru (d) respectively. In all cases the early evolution of the amplitudes and feedthrough from one interface to another is well represented by the linear coupled theory. At late times bubbles 
and spikes diverge and the linear theory greatly overestimates the inner amplitude $\eta_{1}$ in all cases, as expected (for a comparison between the linear and nonlinear regimes in planar RM experiments see Ref. 26. From the explicit expressions in Ref. 27, $\eta \sim t$ in the linear regime and slows down to $\eta \sim \ln t$ in the nonlinear regime).

Snapshots from the 2D CALE simulations are shown in Figs. 8 and 9 for cases (a) and (d) respectively. We do not show snapshots for cases (b) and (c) because they are similar to case (a), Fig. 8, where long gelatin fingers penetrate the central air cavity at late times. It is interesting that the last case, $\eta_{2}(0)=-\eta_{1}(0)=0.5 \mathrm{~mm}$, displays minimum disruption of the inner surface at late times, while the case $\eta_{2}(0)=\eta_{1}(0)=0.5 \mathrm{~mm}$, case (c), displays the most disruption, emphasizing the importance of the relative phases between $\eta_{1}(0)$ and $\eta_{2}(0)$. The analytic theory also shows the correct ordering with case (c) (case (d)) predicted to have maximum (minimum) growth in $\left|\eta_{1}(t)\right|$.

Comparing Fig. 8, case (a), with Fig. 9, case (d), it is interesting to note that adding an initial perturbation with the "right" phase at the inner surface reduces the late-time perturbations at that surface. We hope future experiments will test these predictions.

One of the applications mentioned in the Introduction was ICF capsules. The above gelatin ring experiments help shed light on feedthrough and subsequent growth at the inner surface of single-shell capsules like the ones planned for $\mathrm{NIF}^{8,41}$. Another class of capsules involves double-shell designs ${ }^{8,42}$ where an inner capsule containing the fuel gas is imploded after colliding with an outer shell. To shed light on the many instabilities that can occur in double-shell capsules we have studied the possibility of double-shell gelatin rings. The following system appears to have a wide range of instability growth and is a reasonable experiment to build: $R_{1}=2, R_{2}=2.5, R_{3}=4.5, R_{4}=5.5$, all in centimeters, 
driven by the same oxyacetylene gas. In other words we have taken the original $1.5 \mathrm{~cm}$ thick single shell and broken it up into a $1 \mathrm{~cm}$-thick outer ring and a $0.5 \mathrm{~cm}$-thick inner ring, keeping the outermost surface at $5.5 \mathrm{~cm}$ as before. With atmospheric air trapped in the central cavity and in the annular region between the two shells (i.e. $R_{2}<r<R_{3}$ ), the 1D motion of the shells is shown in Fig. 10.

Note that the implosion is controlled by the outer, heavier shell. It collides with the inner, lighter shell, twice: First at $\sim 550 \mu s$, and a second time at $\sim 700 \mu s$. The first collision drives the inner shell inwards which bounces off the central air and tries to move out, only to be driven in again by the second collision. The outer shell moves out only after that second collision, allowing the inner shell to expand after its second bounce off the central air.

We have studied a large number, but by no means all of the possible combinations of perturbations initiated at one or more interfaces. Of course the mode number can be different at the different interfaces. For brevity we show only 3 cases, all with $n=6$ perturbations of magnitude $0.25 \mathrm{~mm}$. The first two cases have a perturbation at $R_{4}$ or $R_{3}$ only, i.e., at the outer or inner radius of the outermost shell. These are shown in Figs. 11 and 12 respectively. The contrast is striking: The first case hardly disrupts the inner shell, but the second case leads to a large disruption.

We found that the density of the air in the central cavity was a good measure not only of its compression but also the disruption of the cavity. The densities are shown in Fig. 13. During the first bounce $\rho_{1}$ increases to $0.54 \mathrm{~g} / \mathrm{cm}^{3}$ in practically all cases. The second bounce, however, discriminates between the two cases mentioned here: $\rho_{1}$ goes 
up to $0.14 \mathrm{~g} / \mathrm{cm}^{3}$ for the case when $R_{4}$ was perturbed, compared with $0.06 \mathrm{~g} / \mathrm{cm}^{3}$ when $R_{3}$ was perturbed initially. Clearly, more disruption implies poorer compression.

Simulations where only $R_{1}$ or $R_{2}$ was perturbed yielded quite good compressions with $\rho_{1}$ going up to $0.11 \mathrm{~g} / \mathrm{cm}^{3}$ on second bounce. The case where all interfaces were initially perturbed showed poor compression: $0.52 \mathrm{~g} / \mathrm{cm}^{3}$ and $0.07 \mathrm{~g} / \mathrm{cm}^{3}$ on first and second bounce respectively. Fig. 14 shows snapshots of this last case.

\section{Conclusions}

All the results presented in this paper for cylindrical shells have a one-to-one correspondence with their counterparts in spherical shells: Class A and class B solutions, critical modes, geometrical factors, symmetry under $n \rightarrow-n$, etc., occur in cylindrical as well as spherical geometry. It is important to know the details as given here and in Ref. 19 for the purpose of making comparisons or explaining experimental data, but the phenomena are qualitatively the same in both geometries.

As mentioned in the Introduction the advantage of cylindrical geometry lies primarily in ease of diagnostics. The gelatin-ring experiments of Weir et al. are a good example where direct photographs of shell distortions are taken ${ }^{15}$. While it may be possible to prepare and perhaps even implode a hollow gelatin sphere, imaging the inner (and more interesting) surface will be extremely difficult.

We conclude that cylindrical gelatin-ring experiments can exhibit a variety of feedthrough phenomena. Diagnostics of such large-scale phenomena is relatively straightforward, and serve as tests of highly nonlinear calculations on the evolution of perturbations. In the context of ICF capsule implosions the large-scale perturbations can 
serve as a substitute for studying drive asymmetries. A significant advance in diagnostics, however, is needed to observe the small-scale mix expected to occur even at smooth surfaces. Reliable calculations of such turbulent phenomena require 3D calculations beyond our present capabilities, and remain a challenge for the future.

\section{Acknowledgement}

I would like to thank Sam Weir for sharing experimental results and Bruce Goodwin for early insights into the experimental and computational program. This work was performed under the auspices of the U.S. Department of Energy at the University of California, Lawrence Livermore National Laboratory under Contract No. W-7405-ENG48.

\section{Appendix}

In this appendix, we present four class-B analytic solutions to Bell's equation, valid for arbitrary $n A$.

(i) $R=R_{0} e^{t / T}$, where $T$ represents a time constant and the motion is an explosion (implosion) if $T>0(T<0)$. Note that $\ddot{R}=\frac{1}{T^{2}} R$ is positive in both cases. The solution to Eq. (14) is:

$$
\eta(t)=\eta_{0} \frac{\gamma_{+} e^{\gamma_{-} t}-\gamma_{-} e^{\gamma_{+} t}}{\gamma_{+}-\gamma_{-}}+\dot{\eta}_{0} \frac{e^{\gamma_{+} t}-e^{\gamma_{-} t}}{\gamma_{+}-\gamma_{-}}
$$


where

$$
\gamma_{ \pm}=\frac{1}{T}[-1 \pm \sqrt{n A}]
$$

We may write the solution $\eta(t)$ not as a function of $t$ but as a function of $R$ by using

$$
e^{\gamma_{ \pm} t}=\left(R / R_{0}\right)^{-1 \pm \sqrt{n A}} \text {. }
$$

Note that for $n A=0$ the solution reduces to Eq. (17) and for $n A=1$ (critical modes) to Eq. (18).

(ii) $R=R_{0}(1+t / T)^{1 / 2}$. The solution to Eq. (14) is:

$$
\eta(x)=\eta(0) \cos (\gamma x)+\frac{\eta_{x}(0)}{\gamma} \sin (\gamma x)
$$

where

$$
x=\ln \left(1+\frac{t}{T}\right), \gamma=\frac{1}{2} \sqrt{n A-1}
$$

Bell's equation in terms of $x$ simplifies to

$$
\frac{d^{2} \eta}{d x^{2}}+\frac{n A-1}{4} \eta=0
$$

whose solution is Eq. (A4). Note that $\eta_{x}(0)=\frac{d \eta}{d x}(t=0)=T \dot{\eta}_{0}$.

(iii) $R=R_{0}+\dot{R}_{0} t+\frac{1}{2} g t^{2}, g=$ constant. As in spherical geometry, the most difficult case turns out to be the constant acceleration case. Defining 


$$
x=\frac{1}{2 \alpha}\left(t+\frac{\dot{R}_{0}}{g}+\alpha\right)
$$

where

$$
\alpha^{2}=\frac{\dot{R}_{0}^{2}}{g^{2}}-\frac{2 R_{0}}{g},
$$

Eq. (14) becomes

$$
x(1-x) \frac{d^{2} \eta}{d x^{2}}+4\left(\frac{1}{2}-x\right) \frac{d \eta}{d x}+2(n A-1) \eta=0
$$

The solution to this hypergeometric equation is $F(a, b ; c ; x)$ where

$$
a=\frac{3+\sqrt{1+8 n A}}{2}, b=\frac{3-\sqrt{1+8 n A}}{2}, c=2 .
$$

For the case $\ddot{R}=g=0$ Eq. (16) implies $R^{2} \dot{\eta}=$ constant $=R_{0}^{2} \dot{\eta}_{0}$ and hence the solution is formally identical to that of critical modes, Eq. (18), but here there are no limitations on $n A$, which can assume any value. Instead, the motion is given by

$$
R=R_{0}+\dot{R}_{0} t=R_{0}(1+t / T)
$$

which, when substituted in Eq. (18), gives

$$
\eta(t)=\eta_{0}+\frac{\dot{\eta}_{0} t}{1+t / T}=\eta_{0}+\dot{\eta}_{0} t R_{0} / R
$$


This result will prove useful in our next case. Note that $\eta(t)$ is independent of $n A$ unless $\dot{\eta}_{0}$ depends on it, as indeed it will when the motion is initiated by a shock.

(iv) $\ddot{R}=\Delta \mathrm{v} \delta\left(t-t_{s}\right)$. As Richtmyer ${ }^{5}$ showed, an impulsive acceleration can represent the effect of a shock provided certain modifications are made. We substitute $\ddot{R}=\Delta \mathrm{v} \delta\left(t-t_{s}\right)$ in Bell's equation and integrate over $t_{s_{-}} \leq t \leq t_{s_{+}}$, where $t_{s}=$ time of shock arrival and $t_{s_{ \pm}}$are times immediately before and after $t_{s}$. We find

$$
\dot{\eta}_{+}=\dot{\eta}_{-}+(n A-1) \frac{\eta_{s}}{R_{s}} \Delta \mathrm{v}
$$

where $\dot{\eta}_{-}$and $\dot{\eta}_{+}$are the growth rates immediately before and after the passage of the shock, $\eta_{s}$ is the amplitude at shock arrival time and $R_{s}$ is the radius at that time. The effect of the shock is to change the growth rate $\dot{\eta}$ from $\dot{\eta}_{-}$to $\dot{\eta}_{+}$when the interface velocity changes from $\mathrm{v}_{-}$to $\mathrm{v}_{+}$, and of course $\Delta \mathrm{v}=\mathrm{v}_{+}-\mathrm{v}_{-}$is the jump in velocity. For an initially stationary interface $\dot{\eta}_{-}=\mathrm{v}_{-}=0$.

Interesting phenomena, however, can occur if the interface is not stationary before shock arrival as, for example, in a two-shock system. Eq. (A13) applies for each shock. The first shock will activate $R$ and $\eta$ so that the corresponding velocities $\mathrm{v}_{-}$and $\dot{\eta}_{-}$will be nonzero just before the arrival of the second shock, which may or may not be in the same direction as the first shock. The second shock may stop the motion, i.e., $\mathrm{v}_{+}=\mathrm{v}_{-}+\Delta \mathrm{v}=0$. Eq. (A13) still applies with $\Delta \mathrm{v}=-\mathrm{v}_{-}$and the growth rate will, in general, change and perturbations will evolve on that stationary $\left(\dot{R}_{+}=\mathrm{v}_{+}=0\right)$ interface. 
Perhaps a more interesting phenomenon is "freeze-out" ${ }^{\prime 1}$, i.e. $\dot{\eta}_{+}=0$. Clearly, the right-hand-side (rhs) of Eq. (A13) can vanish with the appropriate choice of $\Delta \mathrm{v}$, for example. In this case, the amplitude $\eta$ will freeze at the value $\eta_{s}$ it had at the arrival time of the second shock. This kind of double-shock freeze-out depends on the wavelength of the perturbation because the rhs of Eq. (A13) depends on $n$. We will not elaborate on the shock timing requirements to produce a freeze-out as we have already done so for planar and spherical geometries and the interested reader can work out the conditions for vanishing $\dot{\eta}_{+}$. Let us just mention that in a two-shock system it is not possible to achieve simultaneously the two phenomena discussed above, i.e. to stop $R\left(\mathrm{v}_{+}=0\right)$ and $\eta\left(\dot{\eta}_{+}=0\right)$ at the same time, though each process by itself is quite feasible.

Assuming a shock arriving at $t_{s}=0$ and $\mathrm{v}_{-}=\dot{\eta}_{-}=0$ we get $\dot{\eta}_{+}=(n A-1) \eta_{0} \Delta \mathrm{v} / R_{0}$. Eq. (A12) simplifies to

$$
\begin{aligned}
\eta(t) & =\eta_{0}\left[1+\frac{(n A-1) \Delta \mathrm{v} t}{R_{0}(1+t / T)}\right] \\
& =\eta_{0}\left[1+(\mathrm{nA}-1)\left(1-R_{0} / R\right)\right],
\end{aligned}
$$

which is Eq. (3b).

It is well known that in plane geometry the $R M$ amplitude undergoes a phase reversal if the shock moves from a heavy to a lighter fluid. Of course the amplitude must first go thru zero and from Eq. (3a), this "zero-time" is

$$
t=-\frac{1}{k A \Delta \mathrm{v}}
$$


requiring $\Delta \mathrm{v} A<0$. In cylindrical geometry $\eta=0$ at a time when, from Eq. (A14),

$$
R / R_{0}=1-\frac{1}{n A}
$$

requiring an implosion (explosion) if $A>0(A<0)$. These requirements are in complete agreement with our intuitive expectations of when a phase reversal can occur.

In general, shorter-wavelength-perturbations evolve faster than longer-wavelengthperturbations and hence require less time or motion to zero-out before overshooting and growing with the opposite phase. For example, for $A=1$ the $n=10$ mode will go thru zero when the cylindrical or spherical radius shrinks by $\sim 10 \%$. Of course there is no phase reversal if $A=0$ and the growth for any $\mathrm{n}$ is purely geometrical: $\eta(t)=\eta_{0} R_{0} / R$ from Eq. (A14). 


\section{$\underline{\text { References }}$}

${ }^{1}$ Lord Kelvin, Mathematical and Physical Papers, Hydrodynamics and General Dynamics, IV, (Cambridge, England, 1910).

${ }^{2}$ H. Helmholtz, "Ueber discontinuirliche Flüssigkeitsbewegungen," Wissenschaftliche Abhandlungen 146, J. A. Barth, Leipzig, 1882, translated in Phil. Mag. Ser. 4, 36, 337 (1868).

${ }^{3}$ Lord Rayleigh, Scientific Papers, 2, ( Dover, New York, 1965).

${ }^{4} \mathrm{G}$. I. Taylor, "The instability of liquid surfaces when accelerated in a direction perpendicular to their planes, I,” Proc. R. Soc. London Ser. A 201, 192 (1950).

${ }^{5}$ R. D. Richtmyer, "Taylor instability in shock acceleration of compressible fluids," Commun. Pure Appl. Math. 13, 297 (1960).

${ }^{6}$ E. E. Meshkov, "Instability of the interface of two gases accelerated by a shock wave," Fluid Dyn. 4, 101 (1969).

${ }^{7}$ M. S. Plesset and C. G. Whipple, "Viscous effects in Rayleigh-Taylor instability," Phys. Fluids 17, 1 (1974).

${ }^{8}$ J. D. Lindl, Inertial Confinement Fusion (Springer, New York, 1998).

${ }^{9}$ G. Berthoud, "Vapor explosions,” Ann. Rev. Fluid Mech. 32, 573 (2000).

${ }^{10}$ B. Fryxell, E. Müller, and D. Arnett, "Instabilities and clumping in SN 1987A. I. Early evolution in two dimensions,” Ap. J. 367, 619 (1991).

${ }^{11}$ M. A. Sweeney and F. C. Perry, "Investigation of shell stability in imploding cylindrical targets," J. Appl. Phys. 52, 4487 (1981). 
${ }^{12} \mathrm{M}$. Legrand and N. Toque, "Interface instabilities occurring during an explosive driven implosion," in Proceedings of the Third International Workshop on the Physics of Compressible Turbulent Mixing, Royaumont, France (1991), p. 9.

${ }^{13}$ W. W. Hsing and N. M. Hoffman, "Measurement of feedthrough and instability growth in radiation-driven cylindrical implosions,” Phys. Rev. Lett. 78, 3876 (1997); N. E. Lanier et al., "Multimode seeded Richtmyer-Meshkov mixing in a convergent, compressible, miscible plasma system," Phys. Plasmas 10, 1816 (2003).

${ }^{14}$ E. E. Meshkov, N. V. Nevmershitsky, V. G. Rogachev, and I. G. Zhidov, “Turbulent mixing development investigation with converging jelly rings," in Proceedings of the Fourth International Workshop on the Physics of Compressible Turbulent Mixing, Cambridge, England (1993), p. 578.

${ }^{15}$ S. T. Weir, E. A. Chandler, and B. T. Goodwin, "Rayleigh-Taylor instability experiments examining feedthrough growth in an incompressible, convergent geometry," Phys. Rev. Lett. 80, 3763 (1998).

${ }^{16} \mathrm{Q}$. Zhang and M. J. Graham, "Scaling laws for unstable interfaces driven by strong shocks in cylindrical geometry,” Phys. Rev. Lett. 79, 2674 (1997), and “A numerical study of Richtmyer-Meshkov instability driven by cylindrical shocks," Phys. Fluids 10, 974 (1998).

${ }^{17}$ G. I. Bell, “Taylor instability on cylinders and spheres in the small amplitude approximation," LA-1321 (1951).

${ }^{18}$ M. S. Plesset, “On the stability of fluid flows with spherical symmetry,” J. Appl. Phys. 25, 96 (1954). 
${ }^{19}$ K. O. Mikaelian, "Rayleigh-Taylor and Richtmyer-Meshkov instabilities and mixing in stratified spherical shells," Phys. Rev. A 42, 3400 (1990).

${ }^{20}$ J. D. Ramshaw, "Simple model for linear and nonlinear mixing at unstable fluid interfaces in spherical geometry," Phys. Rev. E 60, 1775 (1999).

${ }^{21}$ C. Cherfils et al, "Convergent Rayleigh-Taylor experiments on the Nova laser," Phys. Rev. Lett. 83, 5507 (1999); S. G. Glendinning et al., “Ablation front Rayleigh-Taylor growth experiments in spherically convergent geometry," Phys. Plasmas 7, 2033 (2000).

${ }^{22}$ K. O. Mikaelian, "Rayleigh-Taylor instabilities in stratified fluids,” Phys. Rev. A 26, $2140(1982)$.

${ }^{23}$ K. O. Mikaelian, "Rayleigh-Taylor and Richtmyer-Meshkov instabilities in multilayer fluids with surface tension,” Phys. Rev. A 42, 7211 (1990).

${ }^{24}$ Y. Yang and Q. Zhang, "General properties of a multilayer stratified fluids system," Phys. Fluids A 5, 1167 (1993).

${ }^{25}$ Y. Yang, Q. Zhang, and D. H. Sharp, "Small amplitude theory of Richtmyer-Meshkov instability, “ Phys. Fluids 6, 1856 (1994).

${ }^{26}$ C. E. Niederhaus and J. W. Jacobs, Experimental study of the Richtmyer-Meshkov instability of incompressible fluids," J. Fluid Mech. 485, 243 (2003); J. W. Jacobs and V. V. Krivets, "Experiments on the late-time development of single-mode RichtmyerMeshkov instability,” Phys. Fluids 17, 034105 (2005).

${ }^{27}$ K. O. Mikaelian, "Explicit expressions for the evolution of single-mode RayleighTaylor and Richtmyer-Meshkov instabilities at arbitrary Atwood numbers," Phys. Rev. E 67, 026319 (2003). 
${ }^{28}$ D. H. Sharp, “An overview of Rayleigh-Taylor instability,” Physica D 12, 3 (1984).

${ }^{29}$ H. J. Kull, “Theory of the Rayleigh-Taylor instability,” Phys. Rep. 206, 197 (1991).

${ }^{30}$ M. Brouillete, "The Richtmyer-Meshkov instability," Ann. Rev. Fluid Mech. 34, 445 (2002).

${ }^{31}$ K. O. Mikaelian, "Richtmyer-Meshkov instabilities in stratified fluids," Phys. Rev. A 31, 410 (1985). One needs to take the thin-shell limit of Eqs. (12) and (13) in this reference. See also "Rayleigh-Taylor and Richtmyer-Meshkov instabilities in finitethickness fluid layers," Phys. Fluids 7, 888 (1995).

${ }^{32}$ A. W. Cook and P. E. Dimotakis, “Transition stages of Rayleigh-Taylor instability between miscible fluids," J. Fluid Mech. 443, 69 (2001).

${ }^{33}$ K. I. Read, "Experimental investigation of turbulent mixing in Rayleigh-Taylor instability," Physica D 12, 45 (1984); D. L. Youngs, "Numerical simulation of turbulent mixing by Rayleigh-Taylor instability,” Physica D 12, 32 (1984).

${ }^{34}$ D. M. Snider and M. J. Andrews, "Rayleigh-Taylor and shear driven mixing with an unstable thermal stratification," Phys. Fluids 6, 3324 (1994); G. Dimonte and M. Schneider, "Turbulent Rayleigh-Taylor instability experiments with variable acceleration," Phys. Rev. E 54, 3740 (1996) and "Density ratio dependence of RayleighTaylor mixing for sustained and impulsive acceleration histories,” Phys. Fluids 12, 304 (2000).

${ }^{35}$ M. Vetter and B. Sturtevant, "Experiments on the Richtmyer-Meshkov instability of an air/SF 6 interface," Shock Waves 4, 247 (1995); L. Erez et al., "Study of the membrane effect on turbulent mixing measurements in shock tubes," ibid 10, 241 (2000). 
${ }^{36}$ S. H. R. Hosseini and K. Takayama, "Experimental investigation of interaction of converging cylindrical shock waves with co-axial cylindrical gaseous interfaces," in Proceedings of the Seventh International Workshop on the Physics of Compressible Turbulent Mixing, St. Petersburg, Russia (1999), p. 33.

${ }^{37}$ S. Kumar, H. G. Hornung, and B. Sturtevant, "Growth of shocked gaseous interfaces in a conical geometry," Phys. Fluids 15, 3194 (2003).

${ }^{38}$ R. E. Tipton, “A 2D-Lagrange MHD code,” in Megagauss Technology and Pulsed Power Applications, edited by C. M. Fowler, R. S. Caird, and D. J. Erickson (Plenum Press, New York, 1987); R. T. Barton, “Development of a multi-material twodimensional, arbitrary Lagrangian-Eulerian mesh computer program," in Numerical Astrophysics, edited by J. M. Centrella, J. M. LeBlanc, R. L. Bowers, and J. A Wheeler (Jones and Bartlett, Boston, 1985).

${ }^{39}$ K. O. Mikaelian and S. T. Weir, "Simulations of gelatin-ring experiments," in Proceedings of the Seventh International Workshop on the Physics of Compressible Turbulent Mixing, St. Petersburg, Russia (1999), p. 409.

${ }^{40} \mathrm{~V}$. N. Goncharov et al., "Modeling hydrodynamic instabilities in inertial confinement fusion targets," Phys. Plasmas 7, 5118 (2000); H. Lin, B. C. Storey, and A. J. Szeri, “Rayleigh-Taylor instability of violently collapsing bubbles," Phys. Fluids 14, 2925 (2002); P. Amendt et al., "Modified Bell-Plesset effect with compressibility: Application to double-shell ignition target designs," Phys. Plasmas 10, 820 (2003).

${ }^{41}$ T. R. Dittrich et al, "Capsule design for the National Ignition Facility," Laser and Particle Beams 17, 217 (1999). 
${ }^{42}$ W. S. Varnum et al., "Progress toward ignition with noncryogenic double-shell capsules," Phys. Rev. Lett. 84, 5153 (2000); P. Amendt et al., "Indirect-drive noncryogenic double-shell ignition targets for the National Ignition Facility: design and analysis," Phys. Plasmas 9, 2221 (2002). 


\section{FIGURE CAPTIONS}

FIG. 1. (a) The radii of an "accel/decel" implosion of a cylindrical shell with $R_{10}=25, R_{20}=26$ in arbitrary units. The acceleration $\ddot{R}_{1}$ is -1.5 for $t<4$ and +1.5 for

$t>4 . R_{2}$ is calculated by mass conservation, $R_{2}=\sqrt{R_{1}^{2}+R_{20}^{2}-R_{10}^{2}}$. (b) The evolution of the perturbation for the implosion shown in (a), assuming $\rho_{1}=\rho_{3}=0$ and $n=10$. The solid lines are the exact solutions from Eq. (10). The dashed lines are solutions to the thin-shell equations, Eqs. (20a,b). The initial conditions read $\eta_{1}(0)=\eta_{2}(0)=1$.

FIG. 2. Same as Fig. 1 for a "shock" implosion: $\ddot{R}=\Delta \mathrm{v} \delta(t)$ with $\Delta \mathrm{v}=-2.7$. The initial and final configurations of the shell are the same as in Fig. 1 and, as in Fig. $1, \rho_{1}=\rho_{3}=0$ and $n=10$. The solid lines in (b) are the exact results from Eq. (10), and the dashed lines are from Eqs. (25a,b) which are the solutions to Eqs. $(20 \mathrm{a}, \mathrm{b})$ for $\ddot{R}=\Delta \mathrm{v} \delta(t)$.

FIG. 3. (a) The radius, (b) the radial velocity, and (c) the radial acceleration of a $1.5 \mathrm{~cm}$ thick cylindrical gelatin ring as calculated by CALE for the experimental conditions described in the text.

FIG. 4. Evolution of perturbation amplitudes $\eta_{1}(t)$ and $\eta_{2}(t)$ for case (a) with initial conditions $\eta_{1}(0)=0$ and $\eta_{2}(0)=0.5 \mathrm{~mm}$. The mode number is $n=6$. The continuous line is the result of the linear coupled equations, Eq. (10). The dashed lines are the bubble and spike amplitudes from 2D CALE simulations shown in Fig. 8. 
FIG. 5. Same as Fig. 4 for the case (b) $\eta_{1}(0)=0.5 \mathrm{~mm}, \eta_{2}(0)=0$.

FIG. 6. Same as Fig. 4 for the case (c) $\eta_{1}(0)=\eta_{2}(0)=0.5 \mathrm{~mm}$.

FIG. 7. Same as Fig. 4 for the case (d) $\eta_{1}(0)=-0.5 \mathrm{~mm}, \eta_{2}(0)=0.5 \mathrm{~mm}$. Snapshots in Fig. 9.

FIG. 8. Snapshots of the gelatin-ring implosion, calculated with CALE, for the case (a) $\eta_{1}(0)=0, \eta_{2}(0)=0.5 \mathrm{~mm}$. Snapshots for cases (b) and (c) are similar to this case. The average radial motion was shown in Fig. 3. The evolution of the perturbations is shown in Fig. 4.

FIG. 9. Same as Fig. 8 for the case (d) $\eta_{1}(0)=-0.5 \mathrm{~mm}, \eta_{2}(0)=0.5 \mathrm{~mm}$.

FIG. 10. The motion of $R_{1}, R_{2}, R_{3}$, and $R_{4}$ versus time for gelatin double rings, assuming atmospheric air trapped in the central cavity $\left(0<r<R_{1}\right)$ and in the annulus between the two shells $\left(R_{2}<r<R_{3}\right)$. The outer shell is driven by the same energy deposition as the single-ring experiments. 
FIG. 11. Snapshots of the double-ring implosion for the case $\eta_{1}(0)=\eta_{2}(0)=\eta_{3}(0)=0$, $\eta_{4}(0)=0.25 \mathrm{~mm}$

FIG. 12. Same as Fig. 11 for the case $\eta_{1}(0)=\eta_{2}(0)=\eta_{4}(0)=0, \eta_{3}(0)=0.25 \mathrm{~mm}$.

FIG. 13. The density $\rho_{1}(t)$ of the air trapped in the central cavity of the double-ring calculations: The continuous line refers to the case $\eta_{4}(0)=0.25 \mathrm{~mm}$, all others being zero (see Fig. 11), and the dashed line refers to the case $\eta_{3}(0)=0.25 \mathrm{~mm}$, all others being zero (see Fig. 12). The second-bounce compression for the latter case is reduced by a factor of $\sim 2$.

FIG. 14. Same as Fig. 11 for the case $\eta_{1}(0)=\eta_{2}(0)=\eta_{3}(0)=\eta_{4}(0)=0.25 \mathrm{~mm} . \rho_{1}(t)$ for this case is similar to the dashed line in Fig. 13, reflecting poor compression at second bounce. 

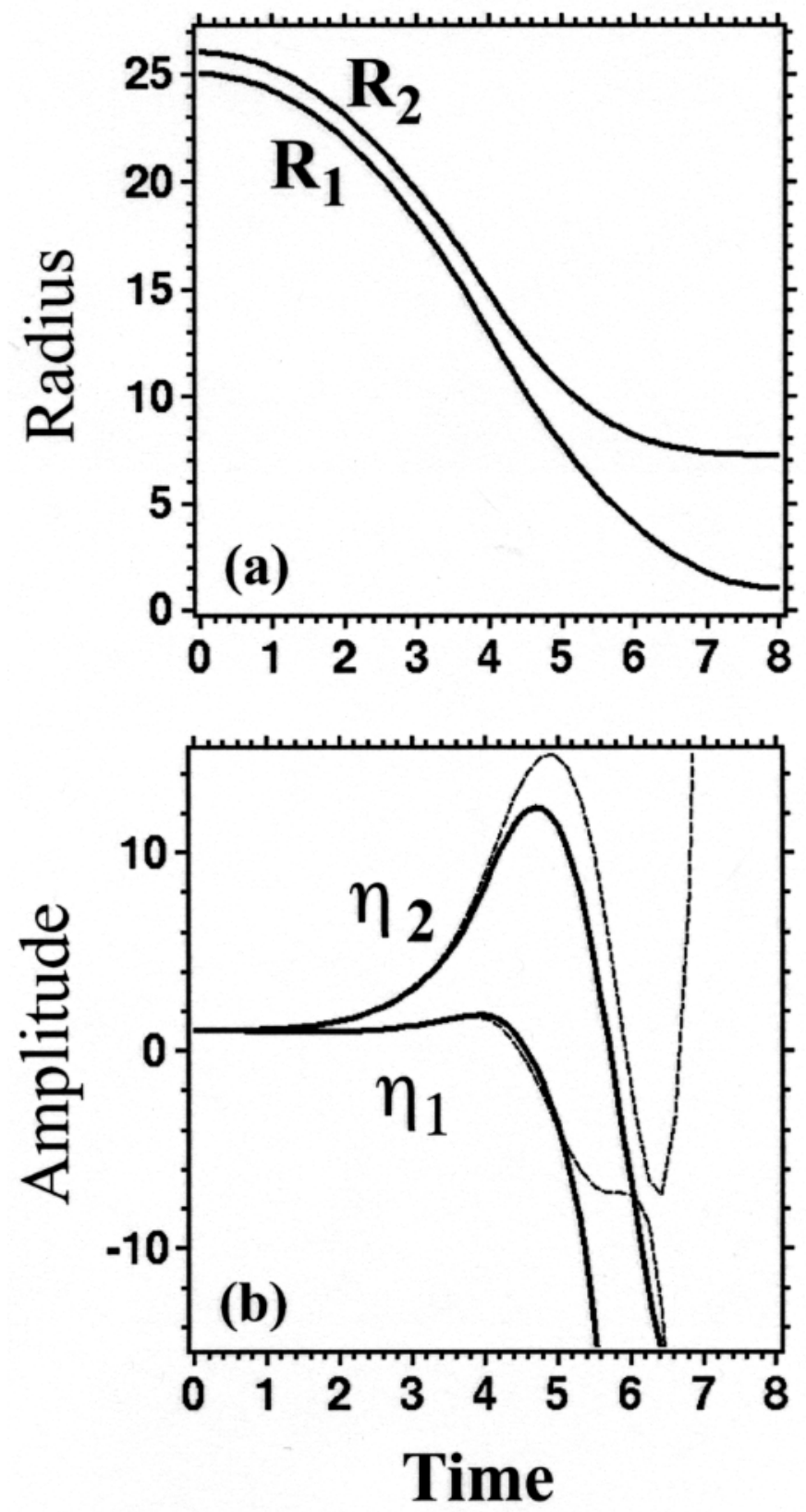

FIG. 1 

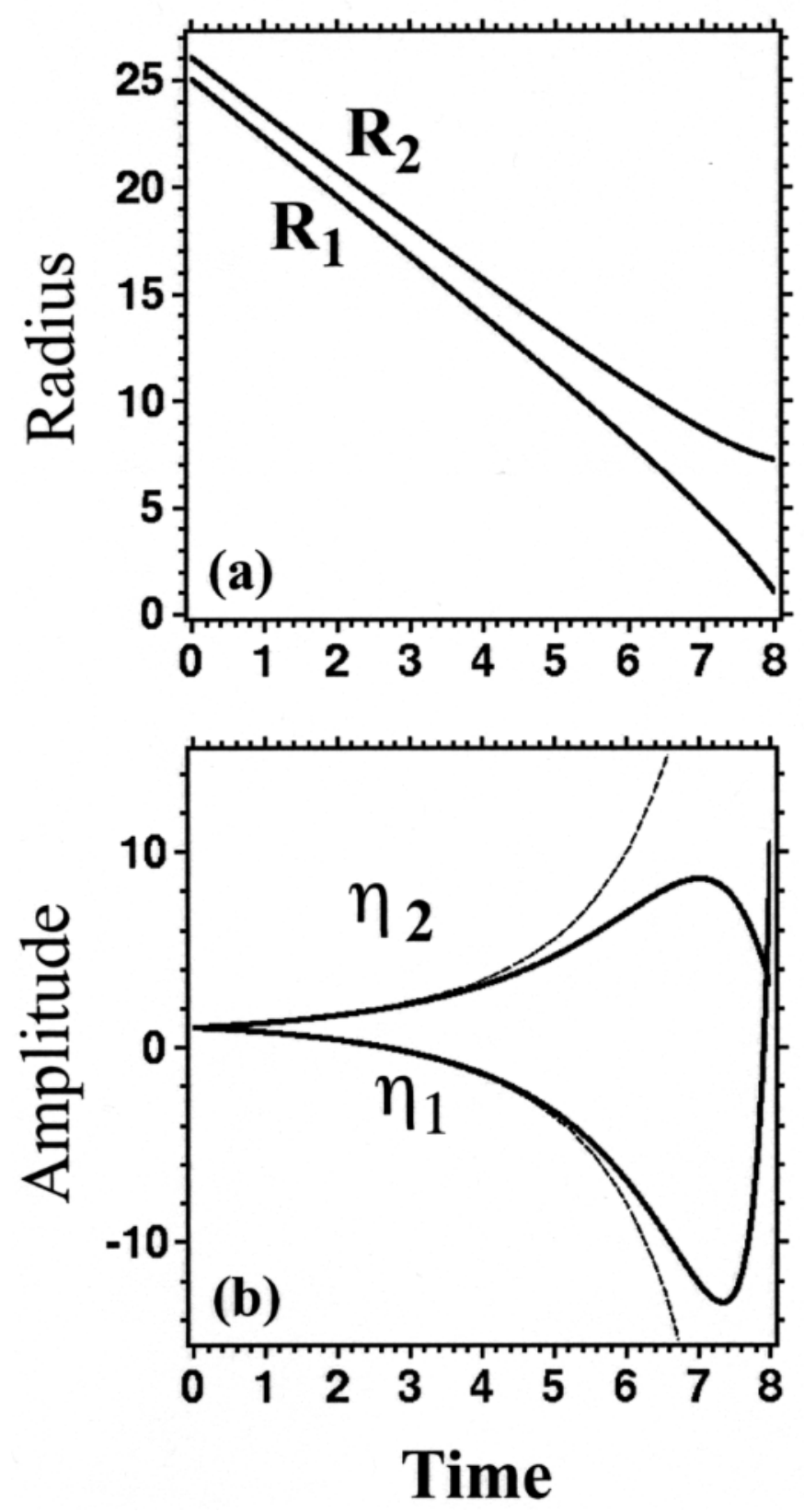

FIG. 2 

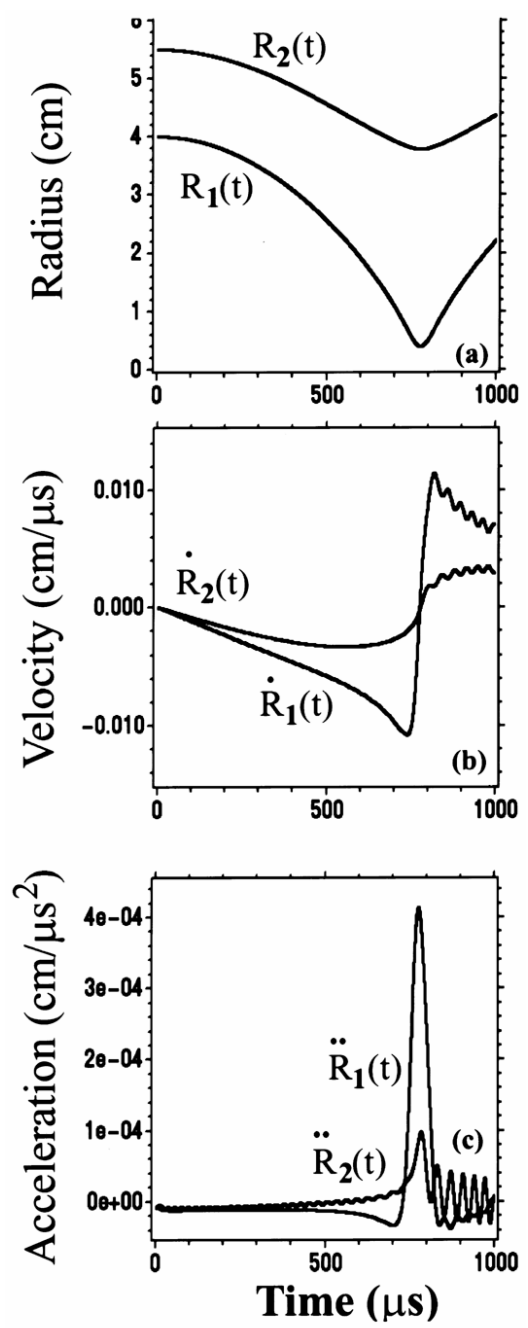

FIG. 3 


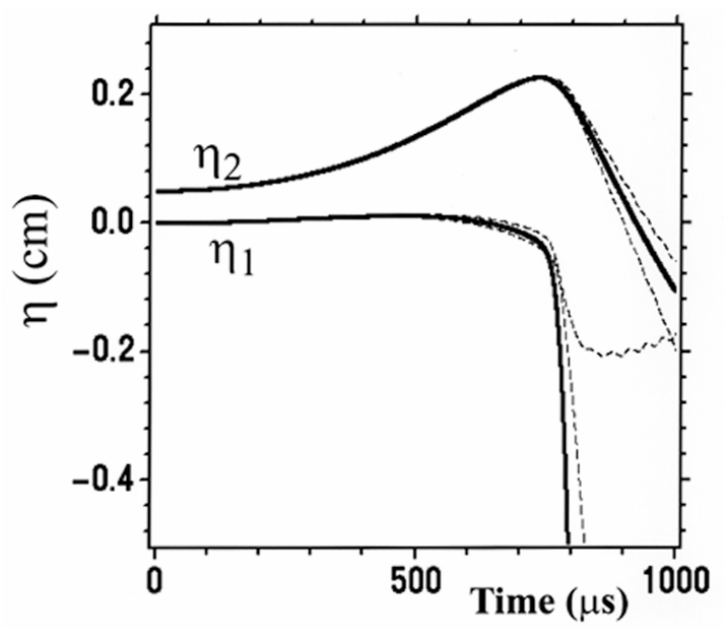

FIG. 4 


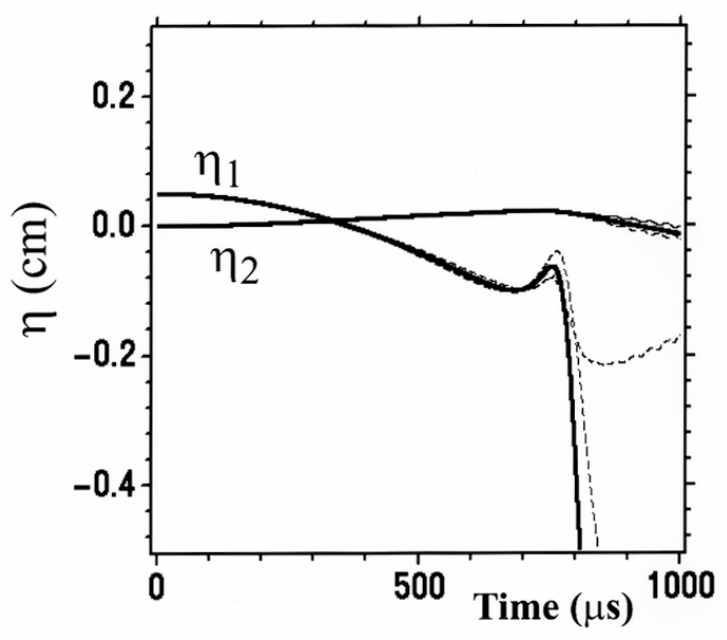

FIG. 5 


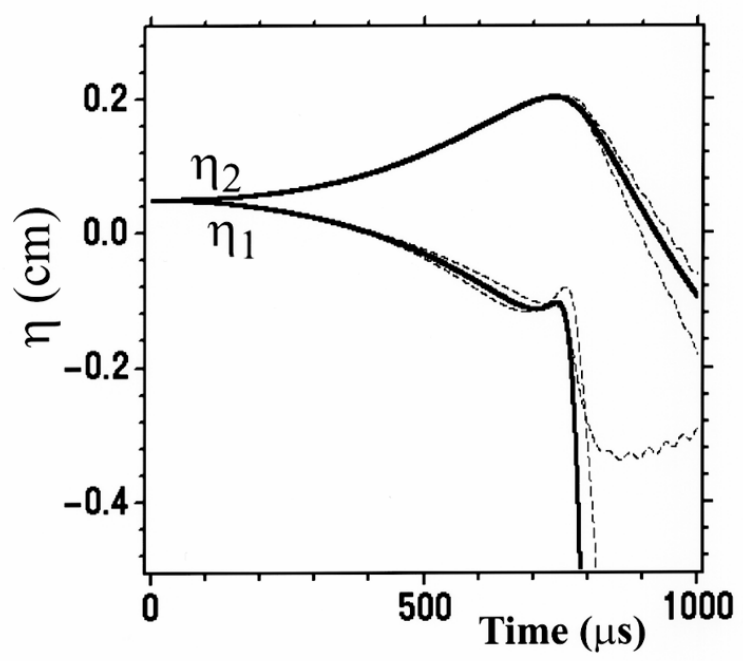

FIG. 6 


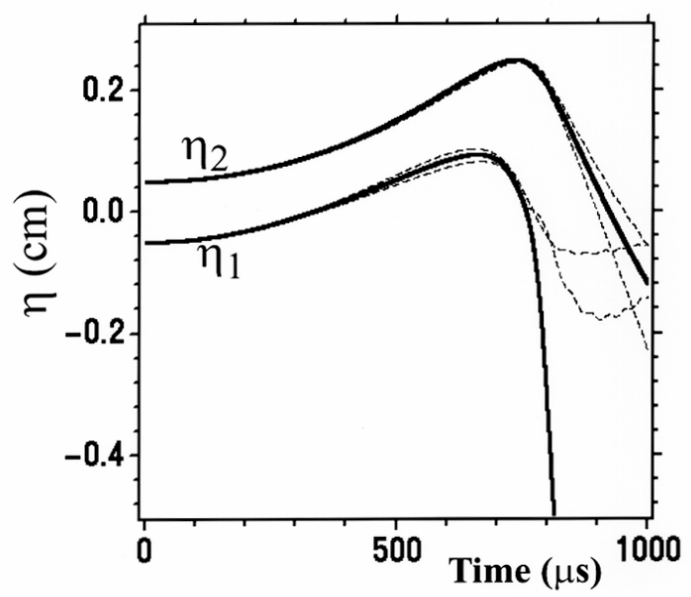

FIG. 7 


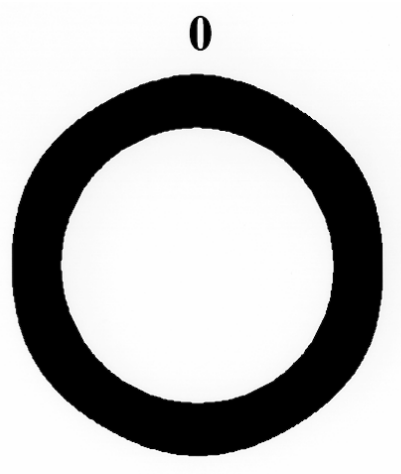

$600 \mu \mathrm{s}$

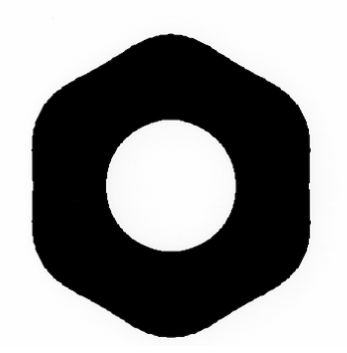

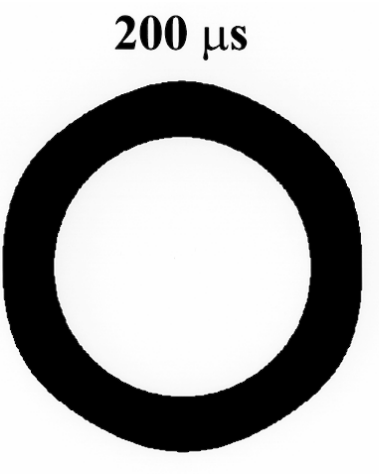

$\mathbf{8 0 0} \mu \mathrm{s}$

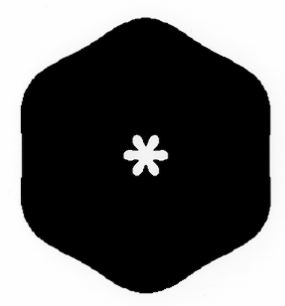

$400 \mu \mathrm{s}$

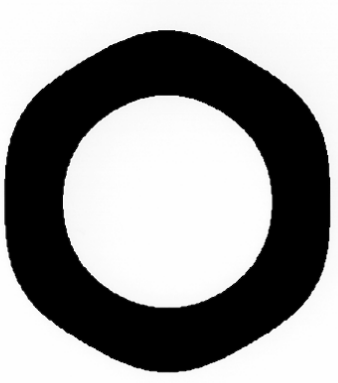

$1000 \mu \mathrm{s}$

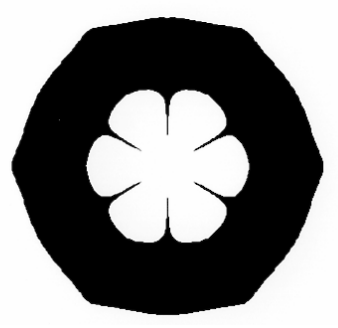

FIG. 8 


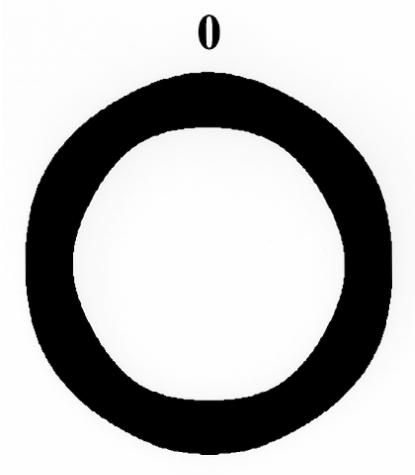

$600 \mu \mathrm{s}$

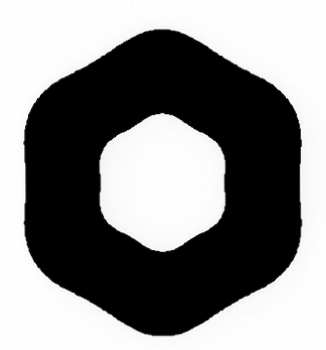

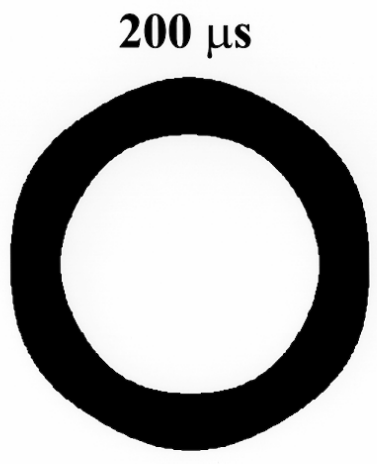

$\mathbf{8 0 0} \mu \mathbf{s}$

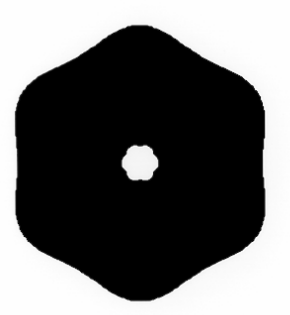

$400 \mu \mathrm{s}$

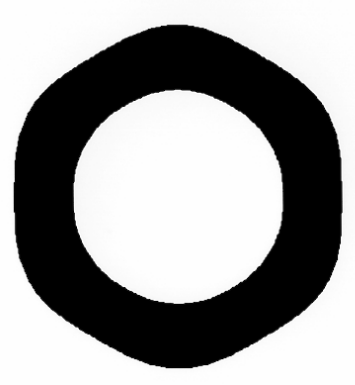

$1000 \mu \mathrm{s}$

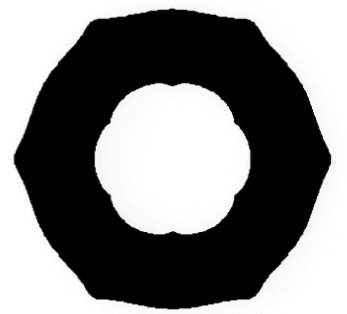

FIG. 9 


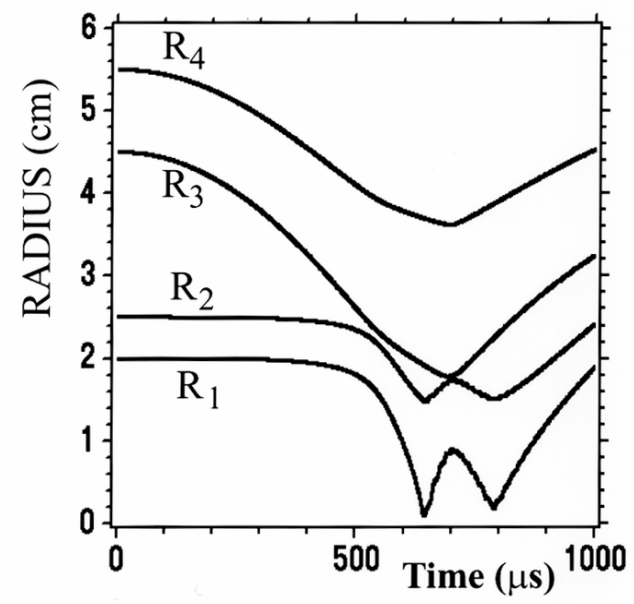

FIG. 10 


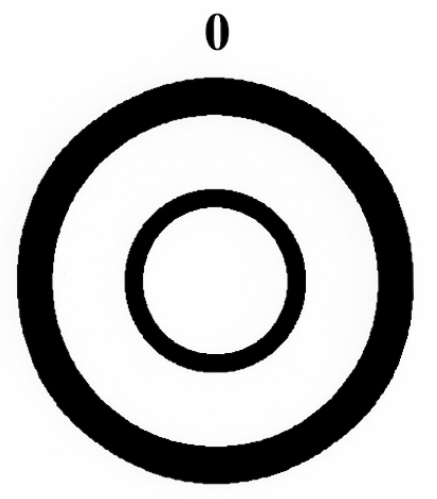

$650 \mu \mathrm{s}$

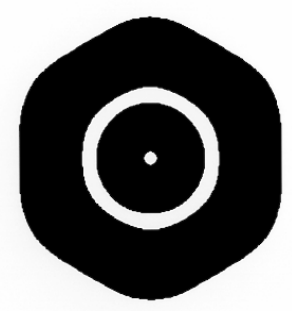

$500 \mu s$

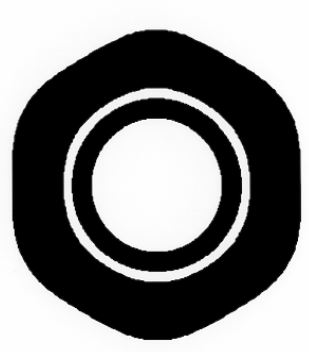

$700 \mu s$

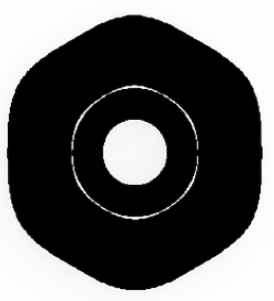

$600 \mu \mathrm{s}$

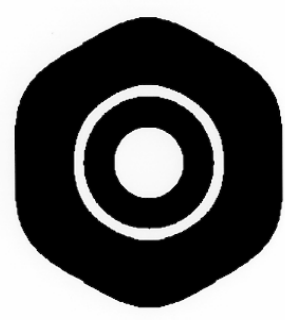

$\mathbf{8 0 0} \mu \mathbf{s}$

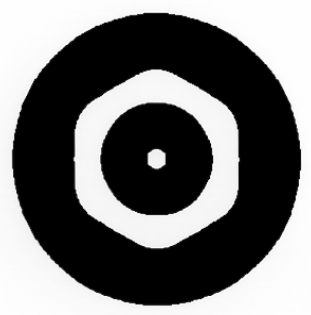

FIG. 11 


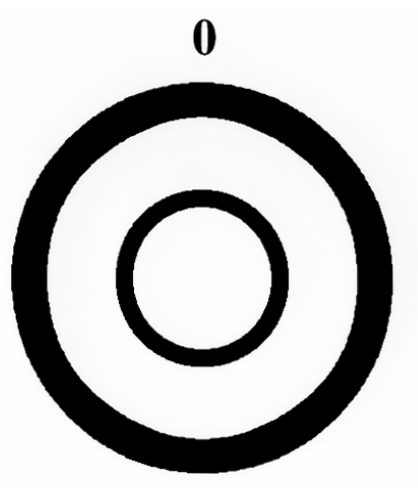

$650 \mu \mathrm{s}$

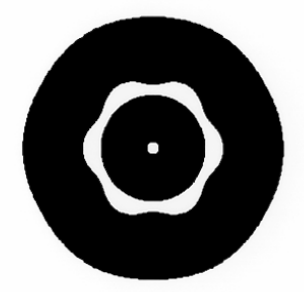

$\mathbf{5 0 0} \mu \mathrm{s}$

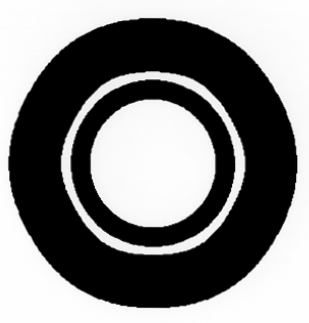

$700 \mu \mathrm{s}$

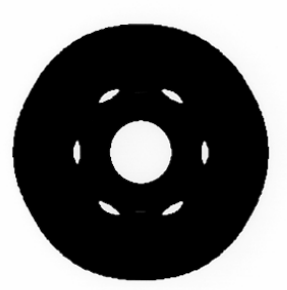

$600 \mu \mathrm{s}$

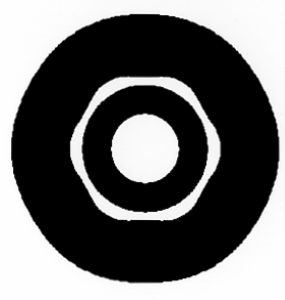

$\mathbf{8 0 0} \mu \mathbf{s}$

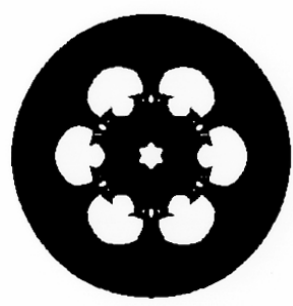

FIG. 12 


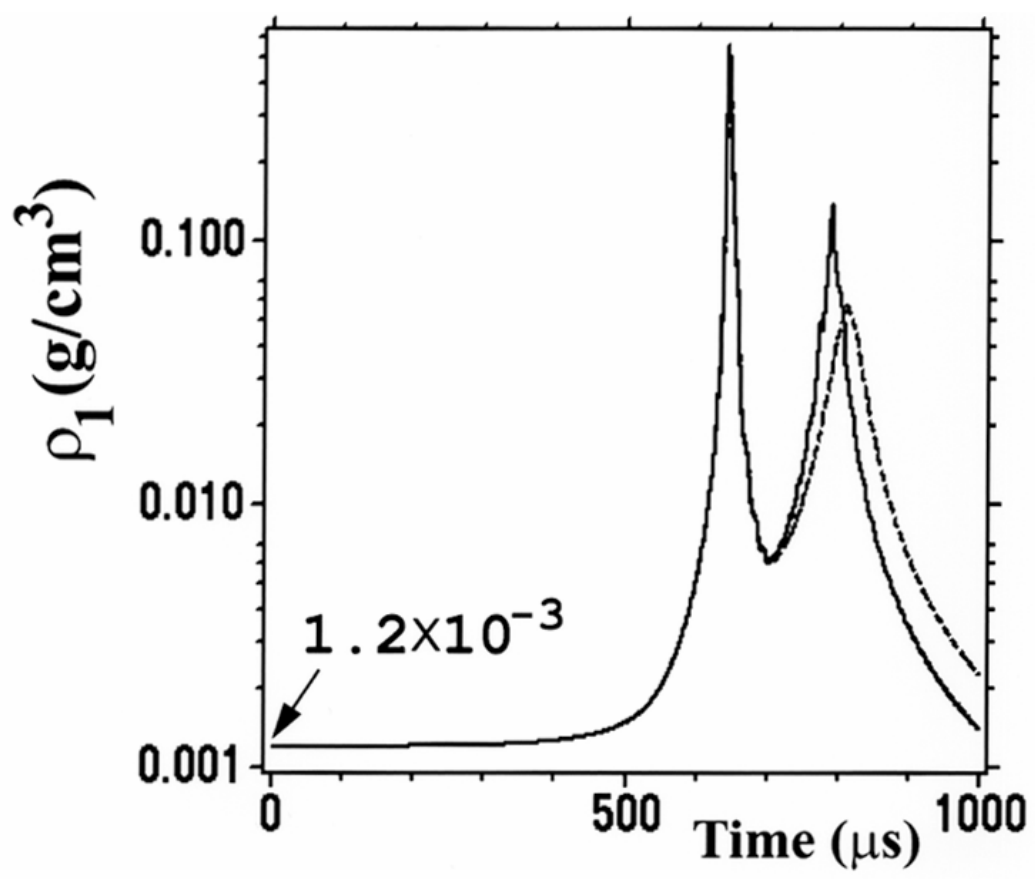

FIG. 13 


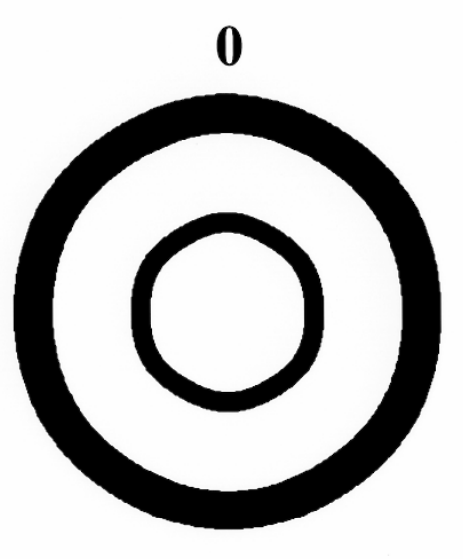

$650 \mu \mathrm{s}$

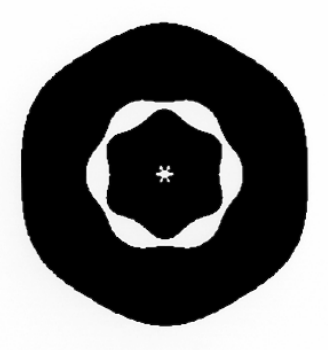

$\mathbf{5 0 0} \mu \mathrm{s}$

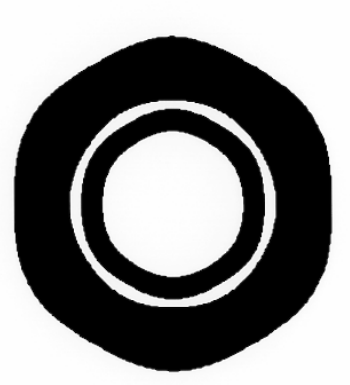

$700 \mu \mathrm{s}$

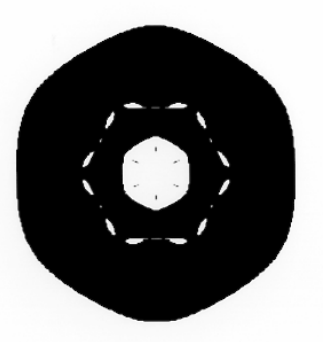

$600 \mu s$

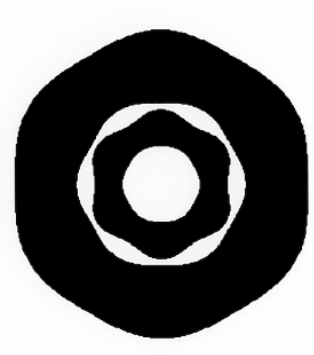

$800 \mu \mathrm{s}$

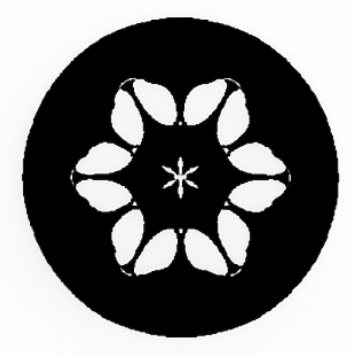

FIG. 14 